\title{
Creep and recovery of magnetorheological fluids: experiments and simulations
}

Article

Accepted Version

Wang, Z., Shahrivar, K. and de Vicente, J. (2014) Creep and recovery of magnetorheological fluids: experiments and simulations. Journal of Rheology, 58 (6). pp. 1725-1750. ISSN 1520-8516 doi: https://doi.org/10.1122/1.4891247 Available at https://centaur.reading.ac.uk/37472/

It is advisable to refer to the publisher's version if you intend to cite from the work. See Guidance on citing.

To link to this article DOI: http://dx.doi.org/10.1122/1.4891247

Publisher: Society of Rheology

All outputs in CentAUR are protected by Intellectual Property Rights law, including copyright law. Copyright and IPR is retained by the creators or other copyright holders. Terms and conditions for use of this material are defined in the End User Agreement.

\section{www.reading.ac.uk/centaur}

\section{CentAUR}

Central Archive at the University of Reading

Reading's research outputs online 


\title{
Creep and recovery of magnetorheological fluids: experiments and simulations
}

\author{
Zuowei Wang, ${ }^{1} *$ Keshvad Shahrivar, ${ }^{2}$ Juan de Vicente ${ }^{2} * *$ \\ ${ }^{1}$ School of Mathematical and Physical Sciences, University of Reading, Reading \\ RG6 6AX, UK \\ ${ }^{2}$ Department of Applied Physics, Faculty of Sciences, University of Granada, C/ \\ Fuentenueva s/n, 18071 - Granada, Spain
}

Corresponding authors:

*zuowei.wang@ reading.ac.uk

**jvicente@ugr.es

\section{Synopsis}

A direct comparative study on the creep-recovery behavior of conventional MR fluids is carried out using magnetorheometry and particle-level simulations. Two particle concentrations are investigated $(\phi=0.05$ and 0.30$)$ at two different magnetic field strengths $\left(53 \mathrm{kA} \cdot \mathrm{m}^{-1}\right.$ and $\left.173 \mathrm{kA} \cdot \mathrm{m}^{-1}\right)$ in order to match the yield stresses developed in both systems for easier comparison. Simulations are mostly started with random initial structures with some additional tests of using preassembled single chains in the low concentration case. Experimental and simulation data are in good qualitative agreement. The results demonstrate three regions in the creep curves: i) In the initial viscoelastic region, the chain-like (at $\phi=0.05$ ) or percolated three-dimensional network (at $\phi=0.30$ ) structures fill up the gap and the average cluster size remains constant; ii) Above a critical strain of $10 \%$, in the retardation region, these structures begin to break and rearrange under shear. At large enough imposed stress values, they transform into thin sheet-like or thick lamellar structures, depending on the particle concentration; iii) Finally in the case of larger strain values either the viscosity diverges (at low stress values) or reaches a constant low value (at high stress values), showing a clear bifurcation behavior. For stresses below the bifurcation point the MR fluid is capable to recover the strain by a certain fraction. However, no recovery is observed for large stress values. 


\section{Keywords}

Magnetorheology, magnetorheological fluids, creep, recovery, unsteady flow, yield stress, yielding, phase diagram.

\section{Introduction}

Magnetorheological (MR) fluids are field-responsive colloidal suspensions that exhibit dramatic changes in their rheological response when magnetized. From a fundamental point of view, they can be used as model systems to explore nonequilibrium transitions from the liquid-state to the solid-state by externally tuning the magnetic field strength. From a practical point of view, there exist currently many commercial applications that involve the use of MR fluids [Ginder (1998); Carlson (2003); de Vicente et al. (2011); Wereley (2013)].

There is nowadays great interest in getting a better understanding of the yielding behavior of MR fluids. In most cases, the yield stress is determined by measuring steady shear properties of MR fluids subjected to a finite range of shear stresses. In principle, by simply extrapolating the steady data at low shear, it is possible to obtain the yield stress. In practice, the situation is more complex as the result may depend on the amount of time we spend measuring every point in the rheogram (if we are not in steady state), and also on whether we are increasing or decreasing the stress (thixotropy and other time-related effects such as sedimentation).

Similar to what occurs for other complex fluids, the study of the creep flow of MR fluids is of valuable help in understanding the yielding behavior of these materials. A total of four regimes have been distinguished when performing creep-recovery tests on MR fluids depending on the stress level applied [Otsubo and Edamura (1994); Li et al. (2002); See et al. (2004); de Vicente and Berli (2013)]. These regimes can be viewed under the frame of the creep-recovery behavior of non-linear viscoelastic materials (see Figure 1). Initially, at very low stresses, MR fluids exhibit a linear viscoelastic response that is characterized by an instantaneous elastic strain $\gamma_{e}$, a retarded elastic strain $\gamma_{r}$ and a viscous strain $\gamma_{v}$. Here, the creep compliance $J=\gamma / \tau_{0}$ remains constant independently of the stress value $\tau_{0}$. Also, both the instantaneous creep strain $\gamma_{i c}$ $\left(=\gamma_{e}+\gamma_{p}\right.$, with $\gamma_{p}$ the plastic contribution) and the instantaneous recovery strain $\gamma_{i r}$ are equal to the elastic contribution $\gamma_{e}$, i. e., $\gamma_{i c}=\gamma_{i r}=\gamma_{e}$ and $\gamma_{p}=0$ (Figure 2a). Upon increasing the stress, the MR fluid behaves as a non-linear viscoelastic material 
and the retarded elastic and viscous strain decrease (see Figure 2b). At this stage, the plastic contribution to the instantaneous strain grows $\left(\gamma_{i c}>\gamma_{i r}\right.$ as $\left.\gamma_{p}>0\right)$. For even larger stresses the MR fluid behaves as a viscoelastic solid (Figure 2c). Here, the strain is instantaneous and fully plastic, and both retarded and viscous strains are negligible. In this regime, $\gamma_{e}=\gamma_{i r}=0$ and $\gamma_{i c}=\gamma_{p}$. Finally, for stresses larger than the so-called "yield stress", the MR fluid behaves as a plastic fluid with a negligible instantaneous creep compliance $\left(J_{0}=0\right.$ ) and a low viscosity level (small $\left.\eta_{0}\right)$ (see Figure $2 \mathrm{~d}$ ). This stage is characterized by the fact that the recovery is negligible and $\gamma_{i c}=\gamma_{i r}=0$.

In this work we aim to investigate the creep-recovery behavior of MR fluids using rheometry and particle level dynamic simulations. Previous experimental work by de Vicente and Berli (2013) focused on dilute MR fluids and suggested a complex yielding mechanism where field-induced aggregates are formed and broken dynamically under shearing. In this manuscript we will present results for highly concentrated MR fluids as well, in an attempt to compare the yielding behavior between dilute and more concentrated suspensions that are closer to commercial applications. Also, the effect of carrier fluid viscosity and waiting times will be investigated.

Finally, it is important to remark that chain-like micromechanical models that are typically and successfully employed under steady shearing flows, fail when trying to explain the creep-recovery behavior of MR fluids [de Vicente and Berli (2013)]. Because of this, in this manuscript we aim to apply dynamic simulations. It is worth to note that in spite of the fact that particle level dynamic simulations have been extensively used in the literature to predict steady shear properties such as viscosity and unsteady small-amplitude oscillatory shear properties such as viscoelastic moduli, the use of particle level simulations in creep flows has not been done yet in the literature. In this manuscript we will show particle level simulation results on creep-recovery tests to better understand the yielding behavior of MR fluids.

\section{Materials and experimental measurement methods}

MR fluids were formulated by dispersing carbonyl iron microparticles in a silicone oil without additives. Carbonyl iron particles were a gift from BASF SE (grade HQ) and silicone oils were purchased from Sigma-Aldrich (20 $\pm 3 \mathrm{mPa} \cdot \mathrm{s}, 98 \pm 3 \mathrm{mPa} \cdot \mathrm{s}$, $340 \pm 5 \mathrm{mPa} \cdot \mathrm{s}$ and $487 \pm 2 \mathrm{mPa} \cdot \mathrm{s})$. MR fluids were prepared at two different particle concentrations: $5 \mathrm{vol} \%(\phi=0.05)$ and $30 \mathrm{vol} \%(\phi=0.30)$. 
Rheology experiments were conducted in a stress-controlled MCR 501 magnetorheometer (Anton Paar) to explore the yielding behavior of MR fluids in the presence of magnetic fields. A plate-plate geometry (diameter $20 \mathrm{~mm}$ ) was used unless otherwise stated. The gap thickness was 300 microns and the magnetic field was perpendicular to the plates. The temperature of the sample was stabilized at $25^{\circ} \mathrm{C}$ using a circulating fluid bath.

Steady shear flow tests were carried out as described in Segovia-Gutiérrez et al. (2012). Briefly, the experimental procedure is summarized as follows: (a) initially the sample was preconditioned at a constant shear rate $200 \mathrm{~s}^{-1}$ for $30 \mathrm{~s}$, (b) next the suspension was left to equilibrate for $1 \mathrm{~min}$ in the presence of a magnetic field, (c) finally the shear stress was logarithmically increased from $0.1 \mathrm{~Pa}$ at a rate of 10 points per decade. Experiments were repeated at least three times with fresh new samples.

Step stress and recovery tests were also performed under shear. The experimental protocol used is summarized as follows: (a) a preshear was first applied to eliminate shear history effects during $30 \mathrm{~s}$ (shear rate $100 \mathrm{~s}^{-1}$ ); (b) an equilibration step followed at rest in a quiescent state (stress equal to zero), again during $30 \mathrm{~s}$; (c) the magnetic field was suddenly applied for a "waiting time" of $120 \mathrm{~s}$-unless otherwise stated- to promote the field-induced structuration; (d) finally, step stress and recovery tests followed still in the presence of the magnetic field. In a typical assay, a constant shear stress $\tau_{0}$ was applied for a time of $300 \mathrm{~s}$ while the resulting strain was measured. The stress was then removed and the recovered strain was measured for another $300 \mathrm{~s}$. In all cases investigated, the strain was reset to zero at the beginning of the creep test. Again, experiments were repeated at least three times with fresh new samples.

\section{Brownian dynamics (BD) simulation methods}

General algorithm. Upon application of a magnetic field, the particles (diameter $\sigma)$ dispersed in a MR fluid become magnetized. The field-induced magnetic dipole moment on particle $i$ is given as

$$
\vec{m}_{i}=\pi \mu_{0} \mu_{s} \beta \sigma^{3} \vec{H}_{l o c} / 2
$$


where $H_{l o c}$ is the local magnetic field acting on the particle and the value of the contrast factor $\beta=\frac{\mu_{p}-\mu_{s}}{\mu_{p}+2 \mu_{s}}$ depends on the relative permeabilities of the particles, $\mu_{p}$, and the suspension, $\mu_{s}$. For the carbonyl iron particles employed in this work, we can find several expressions in the literature for their relative magnetic susceptibility. For example, experiments by Gorodkin et al. (2009) suggested that the specific magnetic susceptibility of iron particles $\chi_{s}$ is a linear function of the median particle size $D_{50}$ (measured in micrometers) with the empirical form of $\chi_{s}=0.668 D_{50}+0.961$. Hence, for a typical particle size of $1 \mu \mathrm{m}$, using Maxwell-Garnett equation, $\chi_{s}=3 \chi_{p} /\left(3+\chi_{p}\right)$, we get that $\chi_{p}=3.56 \approx 4$ and $\beta \approx 4 / 7$. Another popular expression that properly fits the experimental magnetic properties of carbonyl iron particles is the Frölich-Kennely equation [Bozorth (1993)], which gives

$$
\mu_{p}=1+\frac{\chi_{i}}{1+\frac{\chi_{i}}{M_{S}} H}
$$

with $\chi_{i}=131$ and $M_{s}=1990 \mathrm{kA} \cdot \mathrm{m}^{-1}$ [de Vicente et al. (2005)]. Using this equation the iron particles are expected to possess a much larger permeability and therefore form stronger microstructures than that predicted by Gorodkin et al. (2009). For instance, when simulating MR fluids at $\phi=0.05$ under a magnetic field strength of $173 \mathrm{kA} \cdot \mathrm{m}^{-1}$, the use of the Gorodkin equation in estimating the particle permeability resulted in a yield stress in between $100 \mathrm{~Pa}$ and $250 \mathrm{~Pa}$, while a much larger stress in between $400 \mathrm{~Pa}$ and $1000 \mathrm{~Pa}$ was obtained by using the Frölich-Kennely equation. In this work we will stay with the Frölich-Kennely approach [eq.(2)] because it seems to be more widely used in the literature and also generates yield stress values closer to experimental data as will be seen in the following sections.

The dimensionless equation of motion of particle $i$ can be written in the overdamped situation as [Wang (2000)]

$$
\frac{d \vec{r}_{i}^{*}}{d t^{*}}=\sum_{j \neq i}\left(\vec{F}_{i j}^{\text {mag* }}+\vec{F}_{i j}^{r e p *}\right)+\vec{v}_{S}^{*}\left(\vec{r}_{i}^{*}\right)+B \vec{W}_{i}^{*}\left(t^{*}\right)
$$


where $F^{*}=F / \tilde{F}^{m a g}, r^{*}=r / \sigma$ and $t^{*}=t / \tilde{t}$ with the time scaling factor $\tilde{t}=\frac{3 \pi \eta_{s} \sigma^{2}}{\tilde{F}^{m a g}}=$ $16 \eta_{s} / \mu_{0} \mu_{s} \beta^{2} H_{0}^{2}$. The magnetic interaction force $\vec{F}_{i j}^{m a g}$ between two particles is scaled by $\tilde{F}^{m a g}=\frac{3}{16} \pi \mu_{0} \mu_{s} \beta^{2} \sigma^{2} H_{0}^{2}$. Accordingly the shear stress scales as $\tilde{\tau}=\frac{\tilde{F}^{m a g}}{\sigma^{2}}=$ $\frac{3}{16} \pi \mu_{0} \mu_{S} \beta^{2} H_{0}^{2} \cdot \overrightarrow{v_{S}}\left(\overrightarrow{r_{l}}\right)$ is the flow velocity at the particle position $\overrightarrow{r_{l}}$. The stochastic force $\vec{W}_{l}(t)$ follows a standard Gaussian noise distribution and the coefficient $B$ $\left(=\sqrt{\frac{2 k_{B} T}{\tilde{F}^{m a g} \sigma d t^{*}}}\right)$ measures the ratio between the Brownian and magnetic interaction forces. $d t^{*}$ is the dimensionless time step. In the current work, we took a variable step size scheme in which the value of $d t^{*}$ at each BD step was determined to be the time that took the particle under the largest force in the system to move a distance of $0.05 \sigma$. In addition, $d t^{*}$ was subject to an upper bound that was either $d t_{u}^{*}=5 \times 10^{-4}$ or $1 \times 10^{-3}$, depending on the volume fraction of particles and the external field strength. When $d t^{*}=1 \times 10^{-3}$, the coefficient $B$ takes a value of about 0.241 at $H_{0}=53 \mathrm{kA} \cdot \mathrm{m}^{-1}$ and 0.086 at $H_{0}=173 \mathrm{kA} \cdot \mathrm{m}^{-1}$.

BD simulations were performed in a confined geometry as sketched in Figure 3 where the MR fluids were confined in between two parallel planar hard walls. The bottom wall was fixed, while the upper one was allowed to move in the x-direction, either under constant stress or constant shear rate. The simulation box took a cubic shape with periodic boundary conditions in the two unconfined directions. Since for the sample formulation and magnetic field strengths studied in the current work wall slip was not observed in experiments by running measurements at different gaps in plateplate geometries [de Vicente and Berli (2013)], the non-slipping boundary conditions were applied in simulations. If the center of a particle was at a distance less than $0.55 \sigma$ from the surface of a wall, it was assumed to be stuck on the wall laterally, but allowed to move back to the cell in the normal direction. A detailed study on the impact of different boundary conditions will be left for future work.

The short-range interactions between the particles have been argued to play an important role in determining the rheological behavior of MR fluids [Segovia-Gutierrez et al. (2012); Fernández-Toledano et al. (2014)]. Simulation results in the current work were obtained by using an exponential form of the short-range repulsive forces both in between the particles and between the particles and the walls: 


$$
\vec{F}_{i j}^{r e p *}\left(\vec{r}_{i j}^{*}\right)=\left\{\begin{array}{c}
\exp \left[-\frac{\left(r_{i j}^{*}-1\right)}{R_{0}^{*}}\right]\left(-\vec{e}_{r}\right), j=\text { particle } \\
\exp \left[-\frac{\left(h_{i}^{*}-0.5\right)}{R_{0}^{*}}\right] \vec{n}, j=\text { wall }
\end{array}\right.
$$

where $h_{i}^{*}$ is the distance from the center of particle $i$ to the wall surface and $\vec{n}$ is the unit vector in the normal direction of the wall. The cut-off distance of the particle-particle repulsion was $r_{c u t}=1.5 \sigma$, while that with the wall was $0.55 \sigma$. The steepness of the repulsive forces in eq. (4) is controlled by the dimensionless length scale $R_{0}^{*}$ whose value was taken to be $R_{0}^{*}=0.01$. The resulted sharp repulsive potential closely mimics that of hard spheres and so favors the formation of thick column structures. [Klingenberg (1991); Wang et al. (1997)]. The influence of different types of shortrange interaction forces on the simulation results will be left for a further study.

The magnetic interaction forces between the particles were calculated using the dipole-dipole approximation. We employed the coupled-dipole-moment model to take into account the many-body magnetization effect [Wang et al. (1996)]. In this model the instantaneous dipole moment on each particle is evaluated using the local magnetic field at its center:

$$
\vec{H}_{l o c}=\vec{H}_{0}+\sum_{j \neq i} \vec{H}_{j}
$$

where $\vec{H}_{j}$ is the dipolar magnetic field generated by particle $j$ at the center position of $i$. The magnitude of the mutual magnetization effect can be quantified by the mean-square strength of the dipole moments:

$$
\lambda\left(t^{*}\right)=\frac{1}{N} \sum_{1}^{N} m_{i}^{* 2}\left(t^{*}\right)
$$

where $N$ is the total number of magnetic particles in the simulation box and $m^{*}=$ $m /\left(\pi \mu_{0} \mu_{s} \beta \sigma^{3} H_{0} / 2\right) . \lambda\left(t^{*}\right)$ is a time-dependent function that can be used to characterize the change of microscopic structures in the system. Its value also tells to what extent that the coupled-dipole-moment model can improve the quantitative 
description of the magnetic interaction forces over the generally used fixed dipole moment approach where $\lambda=1$ due to the approximation of $H_{l o c}=H_{0}$.

In addition to the mean-square dipole moment strength $\lambda$ [eq. (6)], we introduce two more sets of physical quantities to characterize the structural changes in the system. One is the weight-average size of the field-induced clusters of particles,

$$
S_{\text {avg }}=\frac{\sum n_{i} s_{i}^{2}}{\sum n_{i} s_{i}}
$$

where $n_{i}$ is the number of clusters consisting of $S_{i}$ particles and $\sum n_{i} S_{i}=N$ is the total number of particles in the system. Two particles are considered to be in the same cluster if their center-to-center distance is not larger than $1.05 \sigma$. In another measurement, we classify the $N$ particles in the system into three groups. The fraction of the particles that belong to clusters which are in contact with both confining walls is termed as $f_{2-e n d s}$. Similarly the fractions of those in clusters contacting with only one wall or no wall are termed as $f_{1-e n d}$ and $f_{0-e n d}$, respectively. These fraction values sum up to unity at each time step. In this work, a cluster is considered to be in contact with a confining wall if the center of any particle in this cluster is at a distance $r \leq 0.55 \sigma$ from the surface of the wall. This criterion is consistent with that used for the non-slipping boundary conditions.

Creep-recovery simulation. In creep experiments a constant step stress $\tau_{0}$ is applied to the upper wall of the confined system at time zero. The creep strain of the system is determined by the balance between the applied stress and the internal stress resulted from the viscous friction of the carrier liquid and the interactions among the magnetic particles. The viscous contribution to the internal stress can be estimated by

$$
\tau_{v i s}(t)=\eta_{s} \dot{\gamma}(t)
$$

where $\eta_{s}$ is the dynamic viscosity of the carrier liquid. Here we have neglected the contributions from the viscous dissipation produced by the flow around the particles and the hydrodynamic interactions among particles, which are predicted to give rise to the Newtonian viscosity of a hard sphere suspension $\eta=\eta_{s}\left[1+\frac{5}{2} \phi+(6 \pm 0.2) \phi^{2}\right]$ at 
low particle concentrations. [Larson (1999); Verberg and de Schepper (1997)] For $\phi=0.05, \eta / \eta_{s} \approx 1.67$, while this ratio is estimated to be around $2.5 \sim 3.5$ at $\phi=0.30$ by using high-concentration expressions for $\eta(\phi)$ [Verberg and de Schepper (1997)]. In the MR systems we studied, the value of $\eta$ can also be affected by other experimental conditions such as the particle shape and the residual interparticle aggregations. As will be described in Sec. 4.2, we take an effective value of the carrier liquid viscosity, $\eta_{s}^{e f f}=c_{s} \eta_{s}$ with $c_{s}$ a constant larger than unity, for mapping the experimental and simulation shear stresses at high shear rates. This approach will to a certain extent compensate the approximation made in Eq. (8). The non-hydrodynamic contribution from the interacting particles can be evaluated by a simple approach

$$
\tau_{H}(t)=-\frac{1}{V} \sum_{i, j(\text { all pairs })}\left\langle F_{i j}^{x}(t)\left(z_{i}(t)-z_{j}(t)\right)\right\rangle
$$

where $F_{i j}^{x}$ refers to the projection of the magnetic and short-range interaction forces between particles $i$ and $j$ along the shear direction and $z_{i(j)}$ are their position coordinates in the magnetic field direction.

Using the stress balance in the system [Doi and Edwards (1986); See and Doi (1992)]

$$
\tau_{0}=\tau_{v i s}(t)+\tau_{H}(t),
$$

the instantaneous strain rate is estimated to be

$$
\dot{\gamma}(t)=\left[\tau_{0}-\tau_{H}(t)\right] / \eta_{s^{\prime}}
$$

which in dimensionless units takes the form of

$$
\dot{\gamma}^{*}\left(t^{*}\right)=3 \pi\left[\tau_{0}^{*}-\tau_{H}^{*}(t)\right] .
$$

The instantaneous viscosity of the MR fluid can then be estimated as

$$
\eta(t)=\tau_{0} / \dot{\gamma}(t)
$$


and the creep strain is

$$
\gamma(t)=\int_{0}^{t} \dot{\gamma}(t) d t
$$

The shear creep compliance $J(t)$ can also be obtained from $J(t)=\gamma(t) / \tau_{0}$. In BD simulations the strain increment is updated by

$$
d \gamma=\dot{\gamma}^{*}\left(t^{*}\right) d t^{*}
$$

using the instantaneous strain rate $\dot{\gamma}^{*}\left(t^{*}\right)$ calculated in eq. (12) at every time step.

The creep-recovery simulation of an MR system was performed in three steps with a constant magnetic field $H_{0}$ applied throughout: a) quiescent state: starting from a configuration with randomly distributed particles, the system was equilibrated for a dimensionless time of $5 \times 10^{5} d t_{u}^{*}$ to allow the formation of field-induced structures; $\mathrm{b}$ ) creep process: a constant step stress $\tau_{0}$ was applied to the upper wall of the system at time $t^{*}=0$ and held for a time duration of $10^{7} d t_{u}^{*}$; c) recovery process: the external stress was suddenly removed and the system was relaxed for another time period of $2 \times 10^{6} d t_{u}^{*}$.

In this work, the total number of magnetic particles was fixed to $N=1000$ and a cubic simulation box was used. The side length of the box and also the gap between the two walls were $L=21.88 \sigma$ for the system with particle volume fraction $\phi=0.05$ and $L=12.04 \sigma$ for $\phi=0.30$, respectively. For convenience of comparing with experiments, the dimensionless simulation time has been mapped to real time by multiplying the time scaling factor $\tilde{t}$ calculated from experimental parameters with the only exception of using the effective carrier liquid viscosity $\eta_{s}^{\text {eff }}$ obtained in Sec.4.2 rather than the experimental value of $\eta_{s}$. For a MR system with $\eta_{s}^{\text {eff }}=2.0 \mathrm{~Pa} \cdot \mathrm{s}$ and $H_{0}=173 \mathrm{kA} \cdot \mathrm{m}^{-1}, \tilde{t}$ is approximately $1.4 \times 10^{-3} \mathrm{~s}$. It means that a typical BD run time of $t^{*}=10^{7} d t_{u}^{*}$ with $d t_{u}^{*}=10^{-3}$ is mapped to a real time of $t \approx 14 \mathrm{~s}$. This is apparently much shorter than that studied in experiments. We will thus keep the comparison between simulation and experimental results at the qualitative level. Nevertheless, as will be seen below, the simulation time window is large enough to 
capture all the interesting creep-recovery behavior of MR fluids. More importantly analyses of simulation results are able to provide microscopic understanding of such behavior that is not directly accessible in experiments, particularly at small time scales (e.g., $t \leq 10^{-2} \mathrm{~s}$ ).

Since the single-particle-width chain model has been widely used as the simplest approach to interpret the rheological behavior of MR fluids, we have performed an extra set of creep-recovery simulations using preassembled single-width chains. The system setup is the same as that sketched in Figure 3. In the initial configurations linear chains each consisting of 22 particles were aligned straightly along the magnetic field (z-) direction so as to bridge the two walls with a gap width of $L=22 \sigma$. In the x-y plane, these chains were arranged into a triangular lattice with the lattice vector lengths determined by the particle volume fraction $\phi=0.05$. Periodic boundary conditions were applied in the $\mathrm{x}$ - and $\mathrm{y}$-directions. A number of step stress values were chosen for comparison with simulations using same set of system parameters but random initial configurations.

One remark to be made is that the viscosity $\eta_{s}$, or more precisely $\eta_{s}^{\text {eff }}$, of the carrier liquid does not enter the dimensionless equation of motion [eq.(3)] or particle interaction forces. It only affects the time scaling factor $\tilde{t}$. If a different carrier liquid viscosity is used in experiment, the simulation time can be simply rescaled by the ratio between the two $\eta_{s}$ values.

\section{Results and discussion}

First, creep-recovery experiments were performed using carrier fluids of different viscosities (98 $\mathrm{mPa} \cdot \mathrm{s}, 340 \mathrm{mPa} \cdot \mathrm{s}$ and $487 \mathrm{mPa} \cdot \mathrm{s}$ ) at a given particle concentration $(\phi=0.30)$ under an external magnetic field strength of $H_{0}=53 \mathrm{kA} \cdot \mathrm{m}^{-1}$. Results demonstrated that the viscosity does only affect the time scale for structuration as demonstrated by the fact that creep curves do actually collapse when plotted as a function of the ratio between time and shear viscosity. This finding is important in order to later compare experimental results and simulation data essentially because the time scale used in simulations does also predict that viscosity does not affect the final structure under shear. We note that the shear stress of the MR system will be affected by the viscosity of the carrier liquid. In our simplified theoretical treatment, this effect is taken into account by including the viscous contribution [eq. (8)] in the calculation of 
the total stress [eq. (10)]. However, the neglect of the hydrodynamic contributions, which also rely on $\eta_{s}$, may lead to an underestimation of the shear stress, together with other approximations made in the theoretical framework. Such effects have been partly taken into account by using an effective carrier liquid viscosity for mapping the simulation results to experimental data. Next we carried out experiments in parallelplate (20 mm diameter, $300 \mu \mathrm{m}$ gap) and cone-plate (20 mm diameter, $2^{\circ}$ ) geometries in order to check the impact of a non-homogeneous shear rate acting on the sheared MR fluids. Experiments were done in a concentrated MR fluid $(\phi=0.30)$ formulated in a silicone oil of $487 \mathrm{mPa} \cdot \mathrm{s}$ at $53 \mathrm{kA} \cdot \mathrm{m}^{-1}$. Experimental results demonstrate that both geometries provide very similar results except in the vicinity of the yielding point. This finding also allows a comparison with simulation data where homogeneous shearing flow is assumed (see Figure 3).

Finally, we also explored the effect of waiting time. The waiting time is defined here as the time elapsed in interval c) (see Sec. 2) when the magnetic field is applied, just after the equilibration step. This is an important point because depending on the material under study, some samples significantly age during the rest period. Three different waiting times were investigated $(10 \mathrm{~s}, 60 \mathrm{~s}$ and $120 \mathrm{~s})$. Experiments were done again in a $\phi=0.30 \mathrm{MR}$ fluid formulated in a silicone oil of $487 \mathrm{mPa} \cdot \mathrm{s}$ at $53 \mathrm{kA} \cdot \mathrm{m}^{-1}$. Experimental results demonstrate that structuration occurs very quickly, in less than 10 $s$, and therefore the effect of the waiting times investigated is negligible except at the yielding point where reproducibility is worse. Again, this permits a comparison with simulation data where structures are annealed prior to the creep test.

Following these tests (summarized in Supplementary Information), we performed experimental and simulation studies on the rheological behavior of two MR systems. In one system the volume fraction of particles and the external field strength are taken to be $\phi=0.05$ and $H_{0}=173 \mathrm{kA} \cdot \mathrm{m}^{-1}$, respectively. In the other case, the particle loading and magnetic field strength were $\phi=0.30$ and $H_{0}=53 \mathrm{kA} \cdot \mathrm{m}^{-1}$, respectively. Field strengths were changed in order for the MR fluids to exhibit a similar "yield stress" value of the order of $400 \mathrm{~Pa}$ and for more easy comparison [Segovia-Gutiérrez et al. (2012)]. All simulation data have been mapped to real time and length scales by using the experimental parameters. But as mentioned above the comparison should still be considered as qualitative rather than quantitative due to many assumptions made in the simulation model, including the simplified theoretical treatment of the magnetic and 
short-range interactions between particles, the neglect of hydrodynamic interactions, the assumption of non-slipping boundary conditions, as well as the limited time window and simulation box sizes. From now on we denote the simulation time as $t_{\text {sim }}$ to distinguish from the true experimental time scales.

\subsection{Creep-recovery process}

\subsubsection{Low particle volume fraction $(\phi=0.05)$}

Figures $4(a, b)$ present the experimental and simulation results on the creeprecovery curves of the low concentration MR system for a wide range of step stresses. The yield stress of such a system was estimated to be $\tau_{y} \approx 400 \mathrm{~Pa}$ in a previous publication by de Vicente and Berli (2013). The classical Bingham plastic model predicts a purely elastic solid behavior for step stresses below the yield stress $\tau_{y}$. However, curves depicted in Figures $4(a, b)$ clearly demonstrate three distinctive regimes. At short times (below $10^{-2} \mathrm{~s}$ in experiments and $10^{-3} \mathrm{~s}$ in simulations) there is an instantaneous increase in the strain $\gamma_{i c}$. For longer times the retarded strain $\gamma_{r}$ increases and finally at long times a state of constant strain rate is achieved. Also important to remark is that the recovered strain is only a small fraction of the instantaneous creep strain in contrast to the behavior of linear viscoelastic materials whose behavior is schematized in Figure 2(a). The incomplete recovery of the strain indicates that the MR fluid behaves as a plastic material [de Vicente and Berli (2013)]. For large stresses the MR fluid is not capable to recover the initial strain.

The creep curves obtained in BD simulations also demonstrate three regimes in response to the applied stress, namely an initial response regime, an intermediate retardation regime and a long-time steady state whose feature is determined by the step stress value. The creep curves obtained at stresses below a critical value around $400 \mathrm{~Pa}$ gradually level off at large times, while those achieved at much higher stresses show a typical viscous flow behavior with a constant strain rate. A noticeable difference between the simulation and experimental creep curves is that the former ones possess an inflection point at intermediate time, especially at high stress values. These creeprecovery behavior are analyzed in detail below in relation to the structural changes in the system. 
Initial response regime. Upon the application of the step stress, both experimental and simulation creep curves show a nearly instantaneous jump in strain which is beyond the resolution of the measurement. After that, all these curves grow in parallel with time, regardless of stress values. We have calculated the instantaneous shear strain rate, $\dot{\gamma}(t)$, by taking the time derivatives of each creep curve contained in Figures 4(a, b). This in turn gives the instantaneous viscosity, $\eta(t)=\tau_{0} / \dot{\gamma}(t)$, and the rate of the creep compliance, $\dot{j}(t)=\dot{\gamma}(t) / \tau_{0}=1 / \eta(t)$. The simulation data on $\eta(t)$ are presented in Figure 5(b). In consistence with the parallel feature of the creep curves, all the $\eta(t)$ data collapse onto a universal curve up to a time scale $t_{\text {sim }} \approx 3 \times 10^{-3} \mathrm{~s}$. The same universal behavior was found for the creep compliances $J(t)$, which means that the MR fluid behaves as a viscoelastic material at early time. The response is in the linear viscoelastic region if $\eta(t)$ and so $j(t)$ are constant. This region however lasts only for a very short period of time $\left(t_{\text {sim }} \leq 10^{-4} s\right)$. Beyond that, the viscoelastic behavior is nonlinear with time-variant $\eta(t)$ and $j(t)$ values. Experiments reported in Figure 5(a) also demonstrate a linear viscoelastic region for real times lower than $10^{-2} \mathrm{~s}$.

Figure 6(a) presents the simulation data on the instantaneous stress $\tau_{H}(t)$ calculated using eq. (9) for four stress values, namely $\tau_{0}=100,250,1000$ and $1500 \mathrm{~Pa}$, respectively. Note that the total internal stress in the system is given by $\tau_{\text {tot }}(t)=$ $\tau_{H}(t)+\eta_{S}^{\text {eff }} \dot{\gamma}(t)$. The results show that the time taken for $\tau_{H}(t)$ to reach its plateau value and so for $\tau_{\text {tot }}(t)$ to match $\tau_{0}$ is roughly the same for all the applied stress values. The good agreement between the simulation data obtained from random and preassembled initial configurations also indicates that the stress building-up time is independent of the detailed structures of the system at the beginning of the creep process. It is in this period of time $\left(t_{\text {sim }}<3 \times 10^{-3} s\right)$ that the MR fluid behaves as a viscoelastic material.

The stress component $\tau_{H}$ is determined by the microstructures formed by magnetic particles. We plot in Figures $7(\mathrm{a})$ and (b) the average cluster size, $S_{\text {avg }}$, and the three wall-contact fraction values, $f_{2-e n d s}, f_{1-e n d}$ and $f_{0-e n d}$, as a function of time for the MR systems under a step stress of $\tau_{0}=250 \mathrm{~Pa}$. The vertical dashed line located in between $t_{\text {sim }}=10^{-3} \mathrm{~s}$ and $10^{-2} \mathrm{~s}$ is introduced as a rough guideline marking the end of the initial response regime. Inside the initial regime, the average cluster size remains constant, indicating that the increase of the stress $\tau_{H}$ is solely due to the 
deformation of the chains or columns formed in the quiescent state. It is interesting to look at the changes of the quantities $f_{2-e n d s}$ and $f_{0-\text { end }}$ in Figure 7(b) where all the preassembled single chains were initially in touch with both walls, giving $f_{2-\text { ends }}=1$ at time $t=0 \mathrm{~s}$ (not shown in the plot due to the use of logarithmic time scale). This fraction value drops to zero almost instantaneously upon the application of the stress. In the meantime, the value of $f_{0-\text { end }}$ jumps from 0 to 1 and remains at 1 for the entire initial regime as well as part of the following retardation regime. The breaking of chain end connection with the walls, rather than in the middle of the chain, is qualitatively different from that observed in electrorheological (ER) fluids. In the latter case, the image dipole moments provide strong bonding energy between the polarized particles and the electrodes, and so the chain breakage tends to occur in the middle part of the chain.

The weak linking between the particle clusters and the walls can also be found in Figure 7(a) from the systems with random initial configurations. There the average cluster size at time zero is $S_{a v g} \approx 33.6$ much larger than the single-width chain length of 22. The polydispersity in the cluster sizes can be visualized in the snapshot on the left panel of Figure 9 (a). In this case the initial values of $f_{2-e n d s}$ and $f_{1-e n d}$ are about 0.4 and 0.6, respectively. Compared with single-width chains, the thicker columns can tolerate larger strain deformation by adjusting the relative positions of the particles without losing the internal connectivity or the contact with the walls. The values of $f_{2-e n d s}$ and $f_{1-e n d}$ thus stay constant for a short period of time, but further shear leads to the drop in $f_{2-e n d s}$ and increase in $f_{1-e n d}$ and $f_{0-e n d}$. There is however no chain breakage, as reflected in the constant value of $S_{a v g}$ in this regime.

In Figure 6(a) the $\tau_{H}(t)$ data of the systems with random initial configurations show relative large fluctuations in comparison with that from the systems with preassembled single chains. At longer times the sensitivity of this stress component to the detailed microstructure can result in a quantitative, but not qualitative, diversity in the creep curves obtained from the MR fluids with the same set of system parameters but different initial configurations. Figure 7(b) shows such an example by comparing the creep curves obtained using random and preassembled initial configurations at a stress value of $\tau_{0}=250 \mathrm{~Pa}$. Similar results have been observed in other stress values studied in our simulations. Therefore larger scale ensemble average and bigger 
simulation box are always desired to get more quantitative results comparable to experiments.

The creep curves in Figure 7 imply that the initial viscoelastic regime ends at a strain value around $10 \%$. To check the generality of this observation, we present in Figure 8 (a) the simulation results on $S_{a v g}, \lambda$ and $f_{2-e n d s}$ as a function of the shear strain for various stress values. The mean-square dipole moment $\lambda$ characterizes the overall structure changes in the system by combining the contributions from the cluster size distribution, partly measured by $S_{a v g}$, and the deformation of these clusters under shear, partly reflected in $f_{2-e n d s}$. Apart from the smallest stresses $\left(\tau_{0} \leq 100 \mathrm{~Pa}\right)$ where the maximum strain is only of a few percentages over the entire creep process, the simulation data on $\lambda$ at all other stress values initially collapse onto the same constant value, which is determined by the structures formed in the quiescent state, up to a critical shear strain of $\gamma_{c}=10 \%$. Below $\gamma_{c}, S_{a v g}$ remains stable except for the cases with smallest stress values. On the other hand, the value of $f_{2 \text {-ends }}$ starts to decrease at smaller $\gamma$ values under larger step stresses. This is reasonable because the chain or column structures need to tilt or deform more strongly under larger stress in order to generate internal stress comparable to the applied one. These observations reveal that independent of the system parameters, the shear-induced structure changes in MR fluids before a critical strain value of $10 \%$ only involve the deformation or reorientation of the chains or columns formed in the quiescent state, in absence of structural coarsening. We note that the critical strain value revealed in the creep simulations is in good agreement with the crossover yield strain reported by Segovia-Gutierrez et al. (2012), which is defined as the strain corresponding to the equality of the storage and loss moduli $\mathrm{G}^{\prime}=\mathrm{G}$ '. $\mathrm{BD}$ simulations [Wang et al. (1997)] and theoretical calculations [Gulley and Tao (1993)] of ER fluids in confined geometry also found a chain breaking behavior at the shear strain of $10 \%$.

The $\lambda$ data obtained under different step stresses deviate from each other right after $\gamma_{c}$. For small stress values such as $\tau_{0}=250$ and $400 \mathrm{~Pa}$, both the $S_{a v g}$ and $f_{2-\text { ends }}$ values begin to increase with the creep strain, which consequently results in the increase of $\lambda$. When referring to schematics in Figure 1, it is reasonable to take the instantaneous strains in these cases as $\gamma_{i c}=10 \%$. At larger stresses the clusters in the system keep roughly the same average size, but experience larger scale tilting or deforming, as 
implied by the continuous decrease of $f_{2-e n d s}$, up to the ending time $\left(t_{\text {sim }} \approx 3 \times 10^{-3} s\right.$ ) of the initial response regime. The nonlinear viscoelastic behavior of the system thus lasts beyond the creep strain of $10 \%$, even though the value of $\lambda$ starts to decrease after $\gamma_{i c}$. In this work, we will determine the initial response regime using the universal ending time as estimated in Figures 5(a) and 6(a), rather than the critical strain value, because the former gives a better specification of the region of viscoelastic behavior.

The creep data in Figure 4(a) seem to suggest that the initial response regime found in simulations $\left(t_{\text {sim }}<3 \times 10^{-3} \mathrm{~s}\right.$, as a qualitative measurement) corresponds to the region of time $t<10^{-2} \mathrm{~s}$ in the experimental curves. In this region the experimental data on the instantaneous viscosity collapse onto a universal curve, which shows a decaying trend at early times [see Figure 5(a)]. But in simulations the $\eta(t)$ data increase with time in the initial response regime. The latter is understood as the result of the decreasing strain rate when the field-induced stress $\tau_{H}$ is approaching to the applied stress value $\tau_{0}$. In experiments, inertial effects are clearly affecting such a short time measurements as the initial strain response is quadratic in time [Ewoltd and McKinley (2007)].

Retardation regime. After the initial viscoelastic regime, the dynamics of the MR system is controlled by the balance between the applied step stress and the stress contributions from the particle interactions and viscous flow. When the step stress value is very low, e. g. $\tau_{0} \leq 400 \mathrm{~Pa}$, the stress contribution $\tau_{H}$ generated by the deformed chain or column structures is comparable to or slightly lower than $\tau_{0}$. The strain rate $\dot{\gamma}(t)$ as determined by eq. (12) becomes small, which corresponds to a fast growth in the instantaneous viscosity $\eta(t)$ in these cases. The structural changes of the system in this regime can be seen in Figure 7(a) for the case of $\tau_{0}=250 \mathrm{~Pa}$ with random initial configuration. The increase of the average cluster size, $S_{a v g}$, in this regime indicates the slow aggregation of the smaller clusters into larger ones under shear. This increase shows a stepwise manner owing to the discrete merging events in the simulation box. The system with preassembled single chains behaves somewhat differently. Figure 7(b) shows that the chain size remains constant for most of the retardation regime before a quick rise at the very end. This is because these chains are well separated in the initial state. It takes them much longer migration time than the smaller clusters to aggregate with others, because the friction coefficients of the clusters are proportional to their 
hydrodynamic sizes. The larger creep strain observed in the preassembled chain system can be directly attributed to the thinner chains in comparison with the system starting from random initial configurations.

The mechanical strengths of the column structures get enhanced with the increase in their thickness. As a consequence they become less tilted to sustain the same magnitude of applied stress. This is reflected in the variation of the value of $f_{2-e n d s}$ which shows an upturn at an intermediate time in this regime, while the values of $f_{1-e n d}$ and $f_{0 \text {-end }}$ show inversed trend. When the stress $\tau_{H}$ produced by the thickened structures is large enough to balance $\tau_{0}$, the creep curve levels off and all the structural parameters reach their plateau values, as can be seen in Figures 4, 7 and 8. The system enters the next relaxation regime. During the period of retardation, the average cluster size in the system with random initial configuration at $\tau_{0}=250 \mathrm{~Pa}$ has increased by a factor of 3 from $S_{a v g}=33.6$ to 99.7 . The growth in the cluster thickness can be visualized in the snapshots in Figure 9(a). For the system with preassembled single chains, there is no chain breaking observed. A fraction of the single chains have merged to form double-strand chains. All the chains in the system have at least one of their ends in contact with the walls again, as indicated by the value of $f_{0-e n d} \sim 0$ at the end of this regime.

Under the action of higher step stresses $\left(\tau_{0} \geq 500 \mathrm{~Pa}\right)$, the chain or column structures are highly deformed and become very unstable under shear. In the cases of $\tau_{0} \geq 1500 \mathrm{~Pa}$, some of the clusters are found to be broken by shear flow, which is quantified by the decrease in the $S_{a v g}$ value in Figure 8(a). More generally, the shear flow forces the columns to change their shapes into thin sheet-like structures oriented in the planes defined by the shear and magnetic field directions. These structures bear less mechanical strength and lower flow resistance in comparison with the thick columns. There is consequently a reduction in the instantaneous stress $\tau_{H}(t)$, which is most evident in Figure 6(a) for the case of $\tau_{0}=1500 \mathrm{~Pa}$. The enlarged difference between $\tau_{0}$ and $\tau_{H}$ leads to the increase in the strain rate $\dot{\gamma}(t)$, as implied by eq. (12). The inflection points in the creep curves and so the peaks in the $\eta(t)\left[\sim \dot{\gamma}(t)^{-1}\right]$ data at these high stress values thus correspond to the sign change of the derivatives of $\dot{\gamma}(t)$ at the crossover of the initial and retardation regimes. Such inflection points are not clearly visible in the experimental creep curves in Figure 4. The reason may lie in that in the 
low concentration system these points are located at very short time scales (below $10^{-2} \mathrm{~s}$ ) which are not accessible by experimental measurements. Figure 5(b) shows that after the peak value $\eta(t)$ obtained at high $\tau_{0}$ decreases with time until reaching a constant value. This is in contrast to the continuous growth of the $\eta(t)$ data at lower stress values, and so results in a viscosity bifurcation behavior. This observation is in good agreement with experimental results in Figure 5(a). The viscosity bifurcation phenomena have been reported traditionally in pasty materials [see de Vicente and Berli (2012) and references therein].

Figure 7(c) presents the time dependence of the creep strain and various structural parameters for the case of $\tau_{0}=1000 \mathrm{~Pa}$. The increase in the average cluster size implies that the small pieces of sheet structures gradually merge into large layer structures. This process can be partly visualized in the snapshots in Figure 9(b). The number and thickness of the layers will depend on the volume fraction of the particles. At $\phi=0.05$ only two single layers are formed at the end of the retardation regime, giving the value of $S_{\text {avg }}=500$. But considering the periodic boundary conditions applied, these layers actually extend infinitely in the shear $(x-)$ direction. The formation of layer or lamellar structures have also been reported in ER and MR fluids under oscillatory shear [Wang et al. (1997); von Pfeil et al. (2002); Carletto and Bossis (2003)] and in ER and MR fluids under steady shear [Cao et al. (2006); Fernández-Toledano et al. (2014)]. One driving force for the formation of such structures is to lower the flow resistance.

It is clear from our experimental and simulation results that the MR system behaves qualitatively differently below and above a critical step stress value. The viscosity bifurcation shown in Figures 5(a) and (b) provides a potential pathway to identify the location of this value, or at least narrow down its possible range. In this dilute MR system, the critical stress, which we will notate as $\tau_{b i f u r}$, is apparently sitting in between 600 and $1000 \mathrm{~Pa}$ in experiments and between 400 and $500 \mathrm{~Pa}$ in simulations. It is believed that this stress value is closely related to the yield stress estimated from steady shear experiments. We will discuss this relationship in more detail below.

Long-time steady state. In simulations the onset of the steady state is marked by the time scale at which the structural parameters, such as $S_{\text {avg }}$ and $\lambda$, reach their plateau values. At step stresses below the critical bifurcation value $\left(\tau_{0} \leq 400 \mathrm{~Pa}\right)$, the increment of the shear strains is basically invisible in the creep curves. The instantaneous viscosity 
grows continuously by several orders of magnitude. This means that the motion of the upper wall is still not fully stopped, although the strain rate is getting extremely small. There is thus an aging effect in the system under low step stress.

At large stress values $\left(\tau_{0} \geq 500 \mathrm{~Pa}\right)$, the system enters a constant strain rate regime. The value of the constant rate increases with the increase of the applied stress. From the simulation data in Figures 5(b), 6(a) and 8(a), we see that this regime begins at earlier time, but larger creep strain, for higher $\tau_{0}$ values. The reason is that under higher stress the particles on average have to travel a longer distance before joining the final stable layer structures. The left panel in Figure 9(c) provides a typical side view of the layer structure. Domains that consist of single chains closely packed into triangular lattices are formed, but there is lack of long-range order in the shear direction. In this regime the upper and lower edges of the two single layers take turns to touch the confining walls. Thus the parameters $f_{0-e n d}, f_{1-e n d}$ and $f_{2-e n d s}$ can only take three discrete values $0,0.5$ and 1.0, see Figure $7(\mathrm{c})$. For example, when $f_{0-\text { end }}=0, f_{1-\text { end }}=$ 0.5 and $f_{2-e n d s}=0.5$, one of the layers is only in contact with one wall, while the other is bridging the two walls.

Recovery process. The recovery behavior of the MR system has strong dependence on the step stress values. As shown in Figure 4(b), there is no strain recovery at stresses above the critical value $\tau_{\text {bifur }}$. After the removal of the stress, the shear-induced deformation of the layer structures can be released locally by reorientation of the individual domains and adjustment of the positions of surrounding particles. So no global elastic force is generated to restore some of the strain. The snapshot on the right panel in Figure 9(c) shows that the domain structures can further develop into larger areas in the absence of the external stress.

The situation is qualitatively different at small stress values. The restoration of the deformed chain or column structures causes an instantaneous recovery of the strain, $\gamma_{i r}$. It is followed by a further recovery of magnitude $\gamma_{r}$ before reaching the final stable state. The second recovery process is associated with the slow rearrangement of the column structures. In Figure 10 we present experimental and simulation results on the instantaneously recovered strain, $\gamma_{i r}$, and the total recovered strain, $\gamma_{i r}+\gamma_{r}$, as a function of the stress value $\tau_{0}$. Together with them are the relative fractions of the recovered strains that are calculated as the ratios between the recovered strains and the 
creep strains achieved right before the removal of the stress. Experiments and simulations are in good agreement. The absolute values of the recovered strains first increase with increasing step stress values, and gradually saturate as $\tau_{0}$ getting close to the critical value $\tau_{\text {bifur. }}$. The saturation value of $\gamma_{\text {ir }}$ is about $5 \pm 1 \%$, while that of $\gamma_{i r}+\gamma_{r}$ is around $12 \pm 1 \%$. It is interesting to note that the total recovered strain is close to the instantaneous creep strain of $\gamma_{c} \approx 10 \%$ as observed in the initial response regime for these low stress values $\left(\tau_{0} \leq 400 \mathrm{~Pa}\right)$. The recovered strains can thus be directly attributed to the viscoelastic property of the system consisting of field-induced chain or column structures.

On the other hand, the fractions of the recovered strains decay with the increase of the applied stress. In the case of $\tau_{0}=50 \mathrm{~Pa}, 31 \%$ of the creep strain is recovered instantaneously and a total of $72 \%$ has been recovered. At $\tau_{0}=400 \mathrm{~Pa}$, these fractions are only $1.1 \%$ and $8.7 \%$, respectively. The relative magnitude of recovered strain becomes negligible as the stress value approaches $\tau_{\text {bifur }}$. These results are again in good agreement with experiments.

\subsubsection{High particle volume fraction $(\phi=0.30)$}

The experimental and simulation results on the creep-recovery behavior of the system with $\phi=0.30$ and $H_{0}=53 \mathrm{kA} \cdot \mathrm{m}^{-1}$ are presented in Figures $4-6,8$ and 10-11 for various step stress values. The creep curves in Figure 4 imply that the creep process of the system can still be divided into three regimes, analogous to that observed in the low concentration system. This is further supported by the instantaneous viscosity data in Figure 5 and the structural parameters in Figure 8(b). In the high particle concentration case, both experimental and simulation creep curves obtained at high stress values show the existence of inflection points, which in experiments locate at time scales above $10^{-2} \mathrm{~s}$.

Figure 8(b) shows that the average cluster size at the onset of creep process is $S_{\text {avg }}=1000$, which is equal to the total number of particles in the simulation box. Taking into account the periodic boundary conditions, this means that all the chains or columns in the system are interlinked to form a three-dimensional (3D) percolated gellike structure. At low stress values $\left(\tau_{0} \leq 150 \mathrm{~Pa}\right)$, the percolated network survives the entire creep-recovery simulation time. The snapshot of such a gel-like structure taken at 
the end of the creep process at $\tau_{0}=150 \mathrm{~Pa}$ is given in Figure 11(a). In our simulation model the short-range interactions between particles are purely repulsive. Thus the network formation is solely due to the magnetic interaction forces. In studying the yielding behavior of MR fluids under steady shear, de Vicente and co-workers (2012) proposed a weak-link mechanism for the systems with particle volume fractions above $10 \%$, in which the interlinks between different clusters are weaker and so broken earlier under shear than the bonds inside the clusters. The particle concentration of $\phi=0.30$ clearly falls into that regime. To understand the microscopic picture of the weak-link mechanism, a key issue is to find the correct short-range interaction potential between particles in the experimental systems. This will be left for later study.

Similar to the system with $\phi=0.05$, the general structural properties of the high concentration system remain unchanged up to a creep strain around $10 \%$, see Figure 8(b). The process of stress building-up in this system is demonstrated in Figure 6(b), where the instantaneous stress curves are much smoother than that in the dilute system in Figure 6(a). The reason is that in the initial response regime there are less structural fluctuations in the gel-like network than in the polydispersed chain or column structures. In the retardation regime, the network structure is relatively stable and the average cluster size stays at the constant value of $S_{a v g}=1000$ at step stresses $\tau_{0} \leq 150$ $\mathrm{Pa}$. But at higher stresses the value of $S_{a v g}$ starts to decrease due to the splitting of the 3D network into lamellar structures with various thicknesses, see Figure 11(b) for an example. This structure transition leads to a reduction in the overall mechanical strength of the system, as reflected in the decrease of the instantaneous stress $\tau_{H}$ in the top set of data points in Figure $6(\mathrm{~b})$ where $\tau_{0}=600 \mathrm{~Pa}$. As a result, a constant rate flow is eventually developed in the system. In this constant rate regime, the lamellar structures still undergo splitting and recombining by exchanging layers over time. The value of $S_{\text {avg }}$ thus jumps in between several discrete values, especially at the highest stresses studied. The right panel of Figure 11(b) presents the side view of a single layer taken from a thicker lamellar structure formed at $\tau_{0}=1000 \mathrm{~Pa}$. Different from the domain formation found in Figure 9(c), the particles here arrange into linear arrays aligning along the shear direction. This difference can be understood from the relatively low magnetic field strength $\left(\mathrm{H}_{0}=53 \mathrm{kA} \cdot \mathrm{m}^{-1}\right)$ in this case. The destruction of the chain or column-like structures along the field direction also weakens the mutual magnetization 
effect among the particles. The plateau values of $\lambda$ in this regime are lower than their initial values at the onset of the creep process.

The experimental and simulation data on the instantaneous viscosity in Figures 5(c) and (d) also demonstrate a bifurcation behavior at a critical stress. Experiments locate this value in between $400 \mathrm{~Pa}$ and $1000 \mathrm{~Pa}$. On the other hand, simulations suggest that the value is located in between $\tau_{0}=150 \mathrm{~Pa}$ and $400 \mathrm{~Pa}$. Only when the step stress is below this critical value, there is a partial recovery of the strain. The absolute values of the instantaneous and total recovered strains are close to that obtained in the low density system with $\phi=0.05$, see Figure 10 . The saturation value of the total recovered strain is again around $10 \%$. The fractions of the recovered strains are relatively high in this case, but still decays towards zero as the stress approaches to the critical value. Experiments and simulation data are again in good agreement.

\subsection{Steady shear}

The critical stress value at which the viscosity bifurcation is observed in creep experiments is considered to be related to the yield stress estimated from steady shear measurements. Therefore we have performed two sets of steady shear experiments and simulations using the same system parameters as studied in the creep-recovery simulations. The time-averaged simulation results on the field-induced stress, $\tau_{H}$ [Eq.(9)], and the total shear stress, $\tau_{H}+\eta_{S}^{e f f} \dot{\gamma}$ as used in Eq.(10), are plotted in Figure 12 as a function of the shear rate $\dot{\gamma}$. For comparison, we have also included the creep simulation data at high step stresses where the strain rates were obtained by linear fitting to the constant rate regime of the creep curves. The creep data are related to the total shear stresses measured in steady shear. As expected, the results obtained from these two different types of simulations collapse onto a universal curve and so can be used together for data analysis.

The shear stress data of MR fluids are generally fitted to the Bingham plastic model, $\tau(\dot{\gamma})=\tau_{y}+\eta_{p} \dot{\gamma}$, to give the yield stress $\tau_{y}$. But as discussed in the Introduction, the results heavily rely on how the experimental measures are carried out. This is one of the main reasons for studying the creep-recovery behavior. Instead of trying to find the exact value of $\tau_{y}$ from the steady shear experiments, we use the concept of Bingham model for estimating the time scaling factor to map the simulation data to real time units. According to this model, the shear stress grows linearly with the 
shear rate, at least in the high $\dot{\gamma}$ region. The slope of the $\tau(\dot{\gamma})$ line is the so-called plastic viscosity $\eta_{p}$ which is determined by the viscosity of the carrier liquid and the particle loading. As shown in Figures 12(a) and (b) by the dashed lines, we fit the experimental data points at high shear rates to the Bingham formula to get the values of $\eta_{p}$, which are found to be $2.0 \pm 0.2 \mathrm{~Pa} \cdot \mathrm{s}$ in both systems. Since the plastic viscosity can also be affected by the residual interparticle aggregation, the similar $\eta_{p}$ values found at different particle concentrations may indicate that the particle clusters are not completely disaggregated even at high shear rates. Experimental evidences on this effect are presented in Figure 12(c) and discussed below. For the purpose of making convenient but not fully quantitative comparison between simulation and experimental data, we consider that all the hydrodynamic and particle aggregation effects have been implicitly included in the $\eta_{p}$ values and so set the effective carrier liquid viscosity $\eta_{s}^{e f f}=\eta_{p}(2.0 \mathrm{~Pa} \cdot \mathrm{s})$ in both systems. By replacing $\eta_{s}$ with $\eta_{s}^{\text {eff }}$ in Eq.(8) and mapping the dimensionless simulation time to real time units by multiplying $\tilde{t}=16 \eta_{s}^{\text {eff }} /$ $\mu_{0} \mu_{s} \beta^{2} H_{0}^{2}$, a reasonably good match between the experimental and simulation shear stresses is achieved in the high $\dot{\gamma}$ region, see Figure 12. The corresponding time scaling factors are $\tilde{t} \approx 1.4 \mathrm{~ms}$ at $\phi=0.05$ and $H_{0}=173 \mathrm{kA} \cdot \mathrm{m}^{-1}$ and $11 \mathrm{~ms}$ at $\phi=0.30$ and $H_{0}=53 \mathrm{kA} \cdot \mathrm{m}^{-1}$. These $\tilde{t}$ values have been used to scale all simulation times presented in the current work. We note that this time mapping approach involves some crude approximations. First of all the viscous friction experienced by the particles, as used in the particle equation of motion [eq.(3)], is mainly determined by the carrier liquid viscosity $\eta_{s}$, rather than the effective value $\eta_{s}^{\text {eff }}$. Secondly the exact value of $\eta_{p}$ depends on the shear rate range used for the fitting. Therefore the horizontal shift of the simulation time scales could not be taken as quantitatively well defined. All comparisons between experimental and simulation results should remain at the qualitative level.

We have performed the Bingham model fitting to the simulation results in Figures 12(a) and (b), which include both the steady-shear and creep simulation data points. The yield stress values are found to be $\tau_{y}=475 \pm 20 \mathrm{~Pa}$ for the system with $\phi=0.05$ and $H_{0}=173 \mathrm{kA} \cdot \mathrm{m}^{-1}$ and $317 \pm 15 \mathrm{~Pa}$ for the system with $\phi=0.30$ and $H_{0}=53 \mathrm{kA} \cdot \mathrm{m}^{-1}$. These values are marked by vertical arrows on the abscissa axis in Figure 10. They fall well into the range of the critical stress as estimated from the viscosity bifurcation data 
in Figure 5. This observation thus supports the intrinsic correlation between the yield stress and the critical stress in creep experiments.

It is evident in Figure 12 that although the $\tau(\dot{\gamma})$ curves obtained in simulations and experiments show qualitatively consistent behavior, the simulation data are significantly lower than the experimental values, particularly in the high concentration case. As mentioned before, this discrepancy can be partially attributed to the simplified simulation model. For the system with $\phi=0.05$ and $H_{0}=173 \mathrm{kA} \cdot \mathrm{m}^{-1}$, the meansquare dipole moment of the particles is found to be $\lambda=3.0 \pm 0.2$. It means that the consideration of the local field correction in eq. (5) has already improved the simulation data by at least a factor of 3 in comparison with the point dipole approximation with fixed field strength $H_{0}$. The mutual magnetization effect is relatively weak in the dense system with $\phi=0.30$ and $H_{0}=53 \mathrm{kA} \cdot \mathrm{m}^{-1}$. The value of $\lambda$ calculated from the gel-like networks in the quiescent state is $1.7 \pm 0.1$, which is apparently smaller than that found in the dilute system consisting of separated chain or column structures. As shown in Figure 11(b), the particles in the shear-induced lamellar structures are arranged into arrays aligning along the shear direction, rather than along the magnetic field direction. As a consequence, the data in Figure 12(b) show that the stress $\tau_{H}$ generated by the magnetic interactions between particles reaches a plateau value around $370 \mathrm{~Pa}$, and then slowly increases at higher shear rates. The total shear stresses in these cases are thus dominated by the viscous contribution $\eta_{s}^{e f f} \dot{\gamma}$. This explains why the Newtonian viscosity obtained from the linear fitting to the Bingham model in this case is basically equal to the effective viscosity of the carrier liquid.

The relatively large difference between the experimental and simulation steady shear data in Figure 12(b) can also be correlated to the facts that particles at this concentration are very close to each other and the field strength is very small. These two circumstances make the short-range interparticle interactions, such as remnant magnetization and/or colloidal forces, to play a key role here. One consequence is that some residual interparticle aggregation may exist even at rest (before shearing the sample), which would necessarily result in an extra-contribution to the shear stress as actually observed. The residual interparticle aggregation is present in most of MR fluid formulations and manifests more clearly at large particle loadings or smaller interparticle distances. Figure 12(c) shows the experimental steady shear stress data obtained from MR fluid with a different formulation where a fraction of $\phi=0.30$ 
magnetic particles are dispersed in polyalphaolefin oil (PAO) with and without 1octanol additive. The additive is introduced to help reducing the short-range attractions and consequently the residual aggregations among the particles. The shear stresses obtained from the MR system with additives are clearly lower than that of its counterpart without additives, which can in part explain the discrepancy between the experimental and simulation data in Figure 12(b). Creep experiments on these systems (not presented) have also shown that at a given applied stress the MR fluid with additives achieves larger steady-state strain values or smaller viscosities due to the suppression of residual interparticle aggregation.

\section{Conclusions}

Independently of the particle loading, three regimes are observed in the creep curves: i) Initial response regime where the systems behave in the viscoelastic region and the average cluster size remains constant. In this region field-induced structures deform and reorientate under shear and the material functions collapse onto a universal curve whatever the stress value; ii) Retardation regime where the system behavior results from the balance between the applied stress and the stress contributions from the particle interactions and viscous flow. In this regime, small clusters begin to aggregate forming larger ones but less tilted with respect to the magnetic field direction. For large enough stresses, structures become unstable under shear and eventually assemble into sheet-like or lamellar structures oriented in the planes defined by shear and magnetic field directions; iii) Long time steady state where structural parameters reach plateau values. In the case of large enough stress values the system enters a constant strain rate regime and viscosity bifurcation occurs.

The recovery behavior strongly depends on the stress level. For low stress levels below the bifurcation value, the MR fluid is capable to recover part of the strain. In this case strain increases with increasing stress and gradually saturate. Instantaneous and total recovered strains are in good agreement between experiments and simulations. For stresses larger than the bifurcation value, the recovery is negligible as a result of irreversible structure rearrangements.

As expected, long time creep simulation data are consistent with steady shear flow. In the case of $\phi=0.05$ MR fluids, the comparison between experiments and simulations is good. However, for $\phi=0.30 \mathrm{MR}$ fluids the agreement is not so good 
because this system is more sensitive to interparticle (remnant and colloidal) forces as the concentration is large and the field strength is low. In general, a good qualitative agreement is found between experiments and simulations.

\section{Acknowledgements}

This work was supported by MINECO MAT 2010-15101, MAT 2013-44429-R projects (Spain), by the European Regional Development Fund (ERDF) and by Junta de Andalucía P10-RNM-6630 and P11-FQM-7074 projects (Spain). 


\section{References}

Bozorth, R. M., "Ferromagnetism, " Wiley - IEEE Press, 1993.

Cao, J. G., J. P. Huang, and L. W. Zhou, "Structure of electrorheological fluids under an electric field and a shear flow: experiment and computer simulation, " J. Phys. Chem. B 110, 11635 (2006).

Carletto, P., and G. Bossis, "Field-induced structures and rheology of a magnetorheological suspension confined between two walls," J. Phys.: Condens. Matter 15, 1437-1449 (2003).

Carlson, D. J., "Critical factors for MR fluids in vehicle systems," Int. J. Vehicle Des. 33, 207-217 (2003).

de Vicente J., and C. L. A. Berli, "Aging, rejuvenation, and thixotropy in yielding magnetorheological fluids," Rheol. Acta 52, 467 (2013).

de Vicente, J., D. J. Klingenberg, and R. Hidalgo-Álvarez, "Magnetorheological fluids: a review," Soft Matter 7, 3701-3710 (2011).

Doi, M., and S. F. Edwards, "The theory of polymer dynamics", Oxford Science Publications (1986).

Ewoldt, R., and G. H. McKinley, "Creep ringing in rheometry or how to deal with oftdiscarded data in step stress tests," Rheology Bulletin 76(1), 4-24 (2007).

Fernández-Toledano, J. C., J. Rodríguez-López, K. Shahrivar, R. Hidalgo-Álvarez, L. Elvira, F. Montero, and J. de Vicente, "Elucidating the origin of the two-step yielding in magnetorheology," Submitted.

Ginder, J. M., "Behavior of magnetorheological fluids," MRS Bulletin, August, 26-29 (1998).

Gorodkin, S. R., R. O. James, and W. I. Kordonski, "Magnetic properties of carbonyl iron particles in magnetorheological fluids," J. Phys.: Conference Series 149, 012051 (2009).

Gulley, G. L., and R. Tao, "Static shear stress of electrorheological fluids, " Phys. Rev. E 48, 2744 (1993).

Klingenberg D. J., "Effects of body forces on electro- and magnetorheological fluids," Appl. Phys. Lett. 86, 104101 (2005).

Klingenberg, D. J., "Simulation of the dynamic oscillatory response of electrorheological suspensions: Demonstration of a relaxation mechanism," J. Rheol. 37, 199 (1993). 
Klingenberg, D. J., F. van Swol, and C. F. Zukoski, "The small shear rate response of electrorheological suspensions. I. Simulation in the point-dipole limit," J. Chem. Phys. 94, 6160 (1991).

Larson, R. G., "The structure and rheology of complex fluids", Oxford University Press, Oxford (1999).

Li, W. H., H. Du, G. Chen, and S. H. Yeo, "Experimental investigation of creep and recovery behaviors of magnetorheological fluids," Materials Science and Engineering A333, 368-376 (2002).

Otsubo, Y., and K. Edamura, "Creep behavior of electrorheological fluids," J. Rheol. 38, 1721-1733 (1994).

See, H., and M. Doi, "Shear resistance of electrorheological fluids under time-varying electric fields," J. Rheol. 36, 1143 (1992).

See, H., R. Chen, and M. Keentok, "The creep behaviour of field-responsive fluids," Colloid Polym. Sci. 282, 423-428 (2004).

Segovia-Gutierrez, J. P., C. L. A. Berli, and J. de Vicente, "Nonlinear viscoelasticity and two-step yielding in magnetorheology: A colloidal gel approach to understand the effect of particle concentration," J. Rheol. 56, 1429-1448 (2012).

Verberg, R. and I. M. de Schepper, “Viscosity of colloidal suspensions,” Phys. Rev. E 55, 3143 (1997).

von Pfeil, K., M. D. Graham, D. J. Klingenberg, and J. F. Morris, "Pattern Formation in Flowing Electrorheological Fluids," Phys. Rev. Lett. 88(18), 188301 (2002).

Wang, Z. W., H. P. Fang, Z. F. Lin and L. W. Zhou "Simulation of field-induced structural formation and transition in electromagnetorheological suspensions," Phys. Rev. E 61, 6837 (2000).

Wang, Z. W., Z. F. Lin and R. B Tao, "Many-body effect in electrorheological responses," Int. J. Modern Phys. B. 10, 1153 (1996).

Wang, Z. W., Z. F. Lin, and R. B Tao, "Structure and viscoelasticity of an electrorheological fluid in oscillatory shear: computer simulation investigation," J. Phys. D: Appl. Phys. 30, 1265 (1997).

Wereley, N. M., "Magnetorheology: Advances and Applications," Royal Society of Chemistry (2013). 


\section{FIGURES}

Figure 1.- Schematics of the creep-recovery behavior of a non-linear viscoplastic material. $\gamma_{i c}$ is the instantaneous (creep) strain, $\gamma_{e}$ is the elastic contribution to the instantaneous strain, $\gamma_{p}$ is the plastic contribution to the instantaneous strain, $\gamma_{r}$ is the retardation strain, $\gamma_{v}$ is the viscous (unrecoverable) strain and $\gamma_{i r}$ is the instantaneous recovery strain.

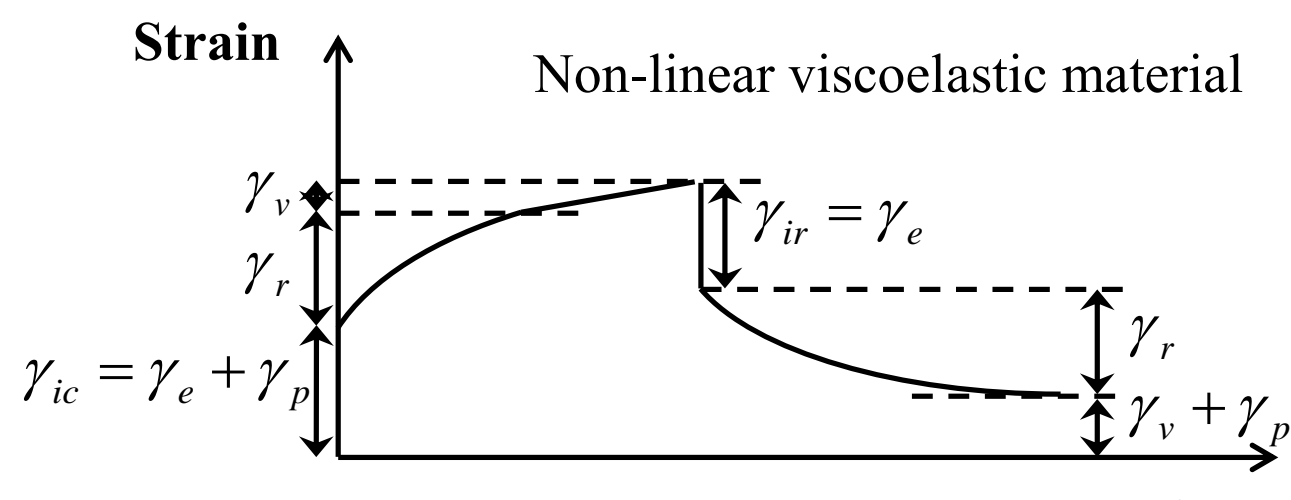

Time 
Figure 2.- Schematics of the creep-recovery behavior of a MR fluid. The stress increases from top to bottom. a) Linear viscoelastic response; b) Non-linear viscoelastic response; c) Viscoplastic solid; d) Plastic fluid.

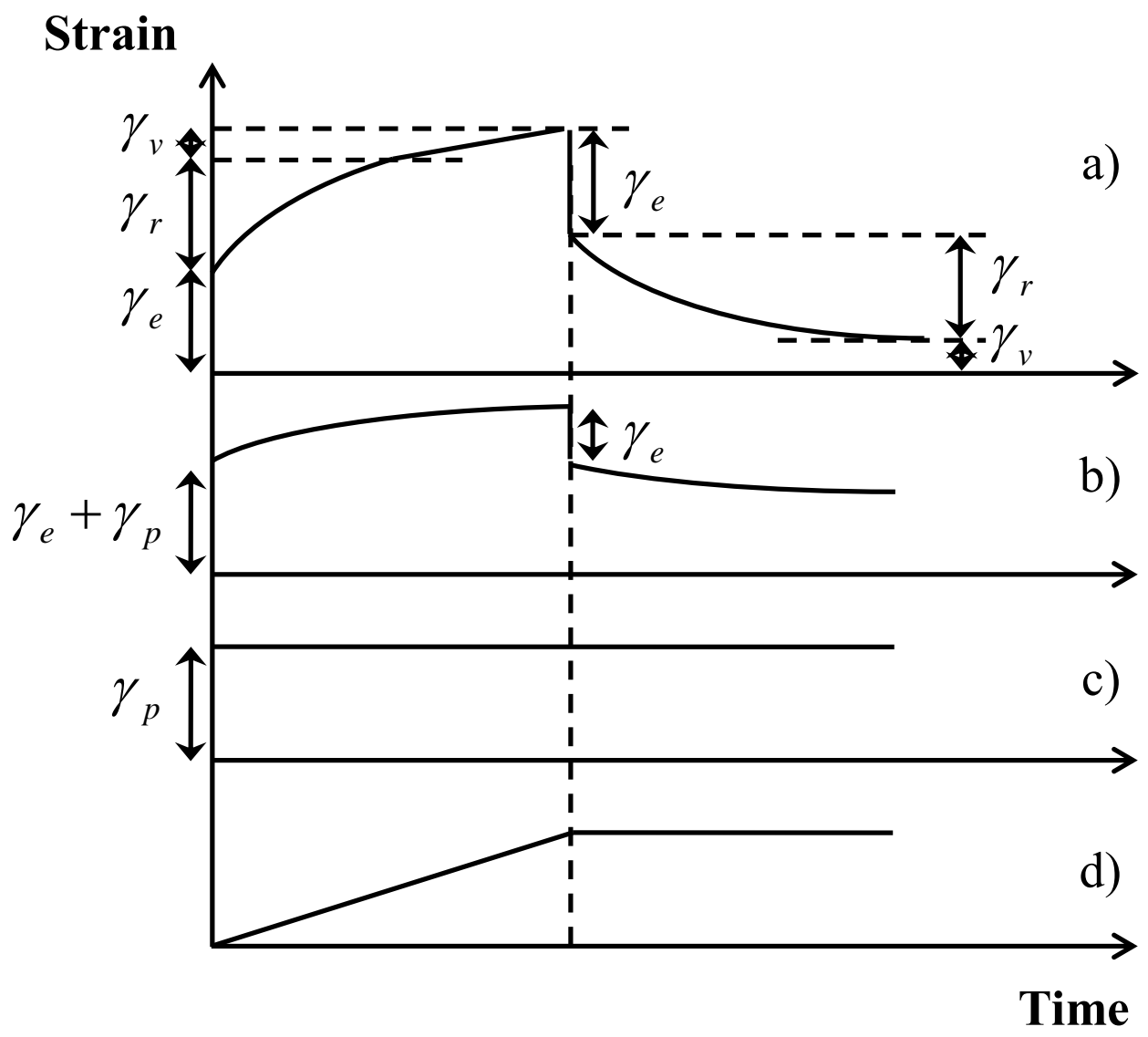


Figure 3.- Sketch of the dynamics simulation system.

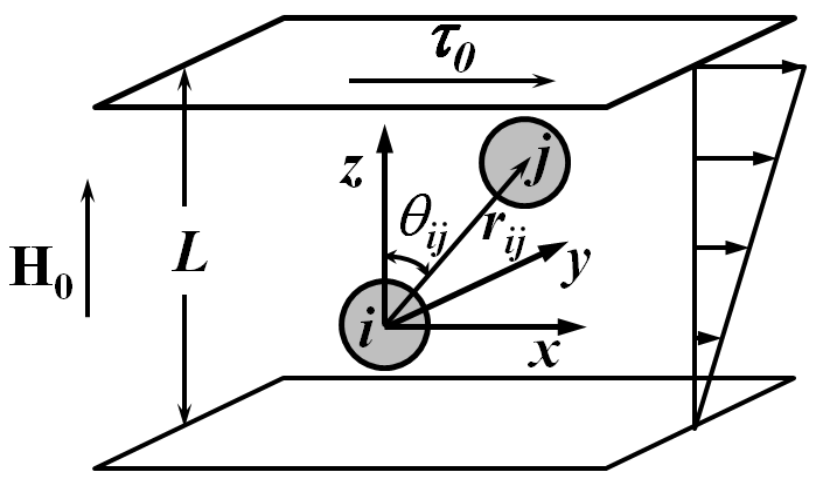


Figure 4.- Creep-recovery curvesobtained at various step stresses as indicated in the graphs for the MR systemswith a) $\phi=0.05 ; H_{0}=173 \mathrm{kA} \cdot \mathrm{m}^{-1} ; \eta_{s}=20 \mathrm{mPa} \cdot \mathrm{s} ; \mathrm{b}$ ) $\phi=0.30 ; H_{0}=53 \mathrm{kA} \cdot \mathrm{m}^{-1} ; \eta_{s}=487 \mathrm{mPa} \cdot \mathrm{s}$. The simulation time scales are calculated using an effective carrier liquid viscosity $\eta_{s}^{\text {eff }}=2.0 \mathrm{~Pa}$.s.

a) $\phi=0.05$; Experiments

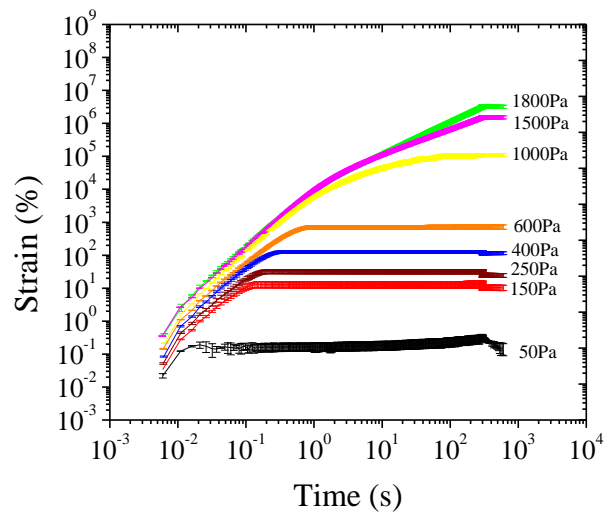

c) $\phi=0.30$; Experiments

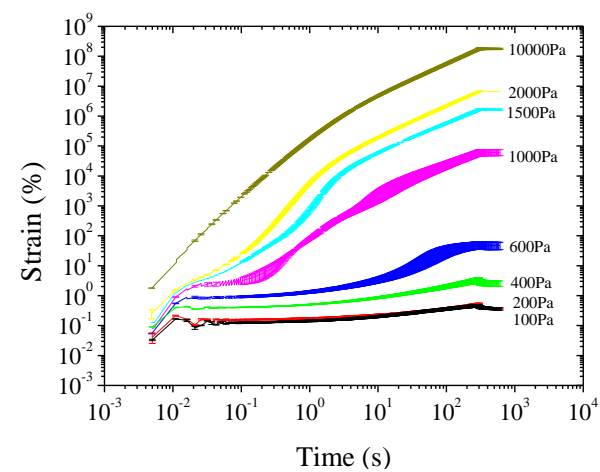

b) $\phi=0.05$; Simulations

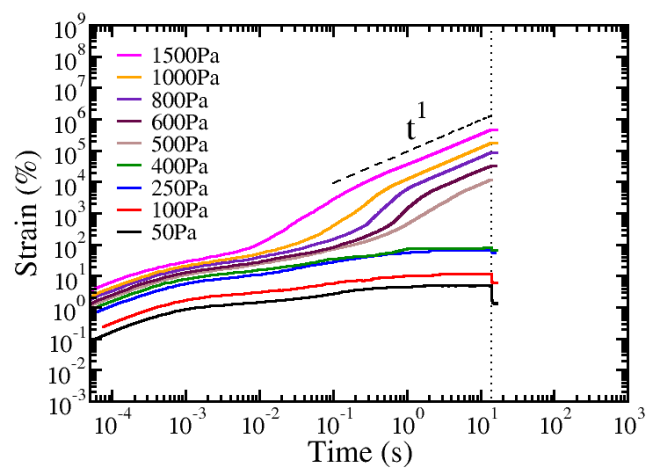

d) $\phi=0.30$; Simulations

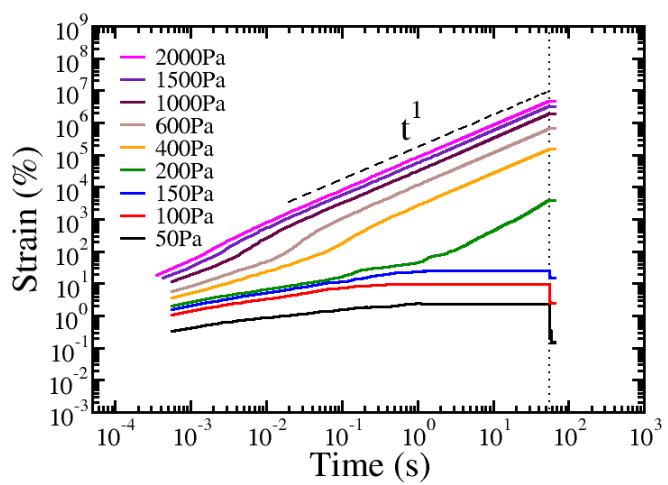


Figure 5.- Instantaneous viscosity as a function of time for the MR systems with a) $\left.\phi=0.05 ; H_{0}=173 \mathrm{kA} \cdot \mathrm{m}^{-1} ; \eta_{s}=20 \mathrm{mPa} \cdot \mathrm{s} ; \mathrm{b}\right) \phi=0.30 ; H_{0}=53 \mathrm{kA} \cdot \mathrm{m}^{-1} ; \eta_{s}=487$ $\mathrm{mPa} \cdot \mathrm{s}$. The results were obtained by taking the time derivatives of the creep strain curves shown in Figure 4. The simulation time scales and instantaneous viscosities are calculated using an effective carrier liquid viscosity $\eta_{s}^{e f f}=2.0 \mathrm{~Pa} . \mathrm{s}$.

a) $\phi=0.05$; Experiments

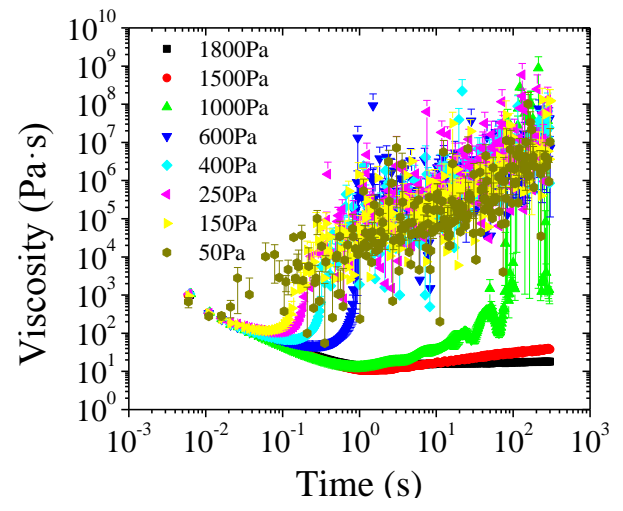

c) $\phi=0.30$; Experiments

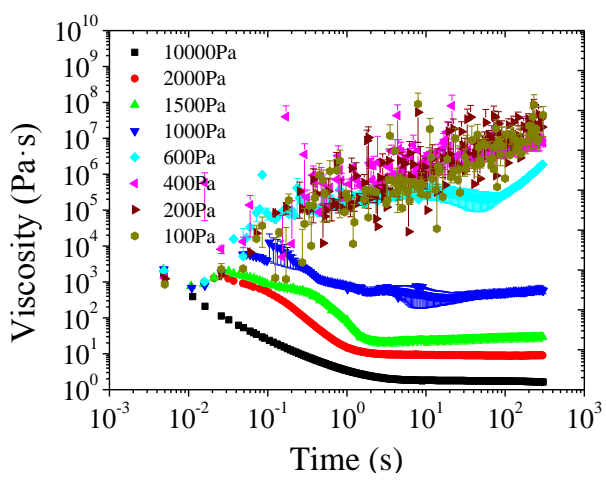

b) $\phi=0.05$; Simulations

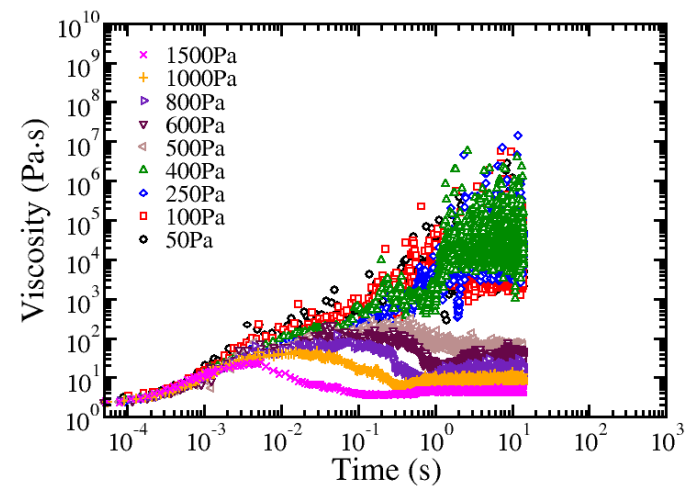

d) $\phi=0.30$; Simulations

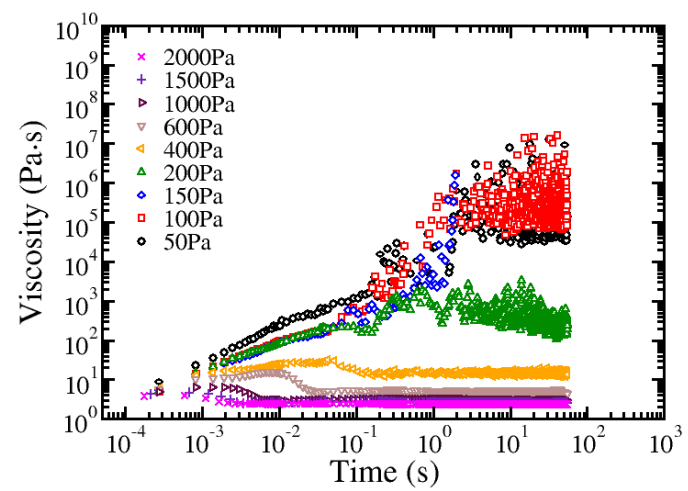


Figure 6.- Building-up of the internal stress $\tau_{H}$ [eq.(9)] in response to the applied external stress $\tau_{0}$ (horizontal lines). The system parameters are: a) $\phi=0.05 ; H_{0}=173$ $\left.\mathrm{kA} \cdot \mathrm{m}^{-1} ; \eta_{s}=20 \mathrm{mPa} \cdot \mathrm{s} ; \mathrm{b}\right) \phi=0.30 ; H_{0}=53 \mathrm{kA} \cdot \mathrm{m}^{-1} ; \eta_{s}=487 \mathrm{mPa} \cdot \mathrm{s}$. Simulation results obtained from random initial configurations are given by symbols, while those from preassembled single-width chains are presented as dashed curves [only in a)]. The simulation time scales are calculated using an effective carrier liquid viscosity $\eta_{s}^{\text {eff }}=2.0$ $\mathrm{Pa} \cdot \mathrm{s}$.

a) $\phi=0.05$

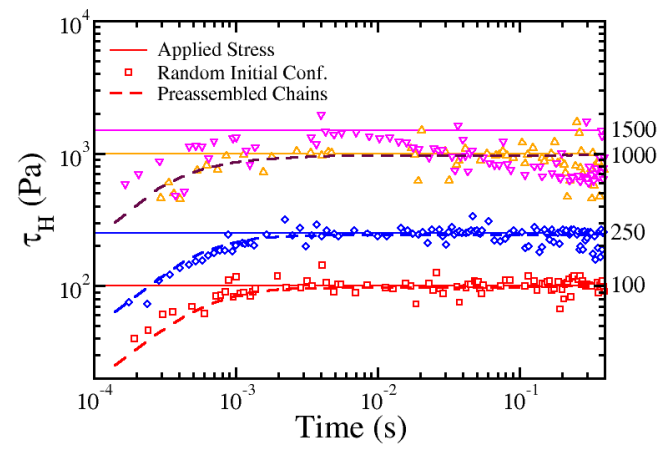

b) $\phi=0.30$

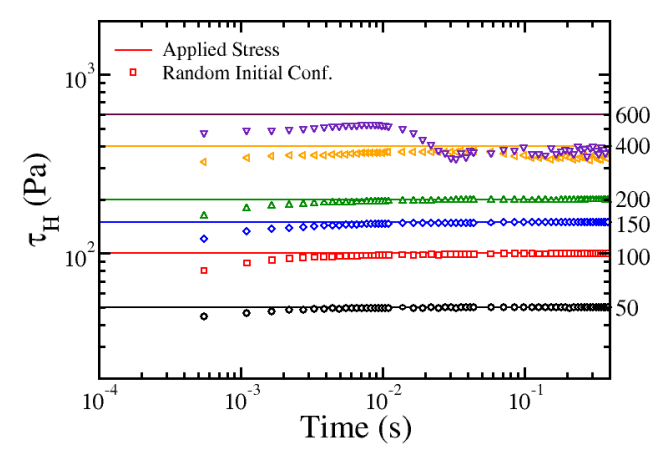


Figure 7.- Shear strain $\gamma$, average cluster size $S_{\text {avg, }}$, and wall-contact fractions parameters $f_{2-e n d s, 1-e n d, 0-e n d}$ (see definitions in main text) as a function of time obtained from simulations starting with random initial configurations (a and c) and preassembled array of single-width linear chains (b). In all plots $\phi=0.05$ and $H_{0}=173$ $\mathrm{kA} \cdot \mathrm{m}^{-1}$. The applied stress is $\tau_{0}=250 \mathrm{~Pa}$ for (a) and (b), and $1000 \mathrm{~Pa}$ for (c). The strain curve obtained in (a) is also plotted in (b) as the dotted-dashed line for comparison. The vertical dashed lines in the plots are qualitative guidelines for dividing the creep process into three dynamic regimes. The simulation time scales are calculated using an effective carrier liquid viscosity $\eta_{s}^{e f f}=2.0 \mathrm{~Pa} \cdot \mathrm{s}$.

a) $\tau_{0}=250 \mathrm{~Pa}$; Random initial configurations

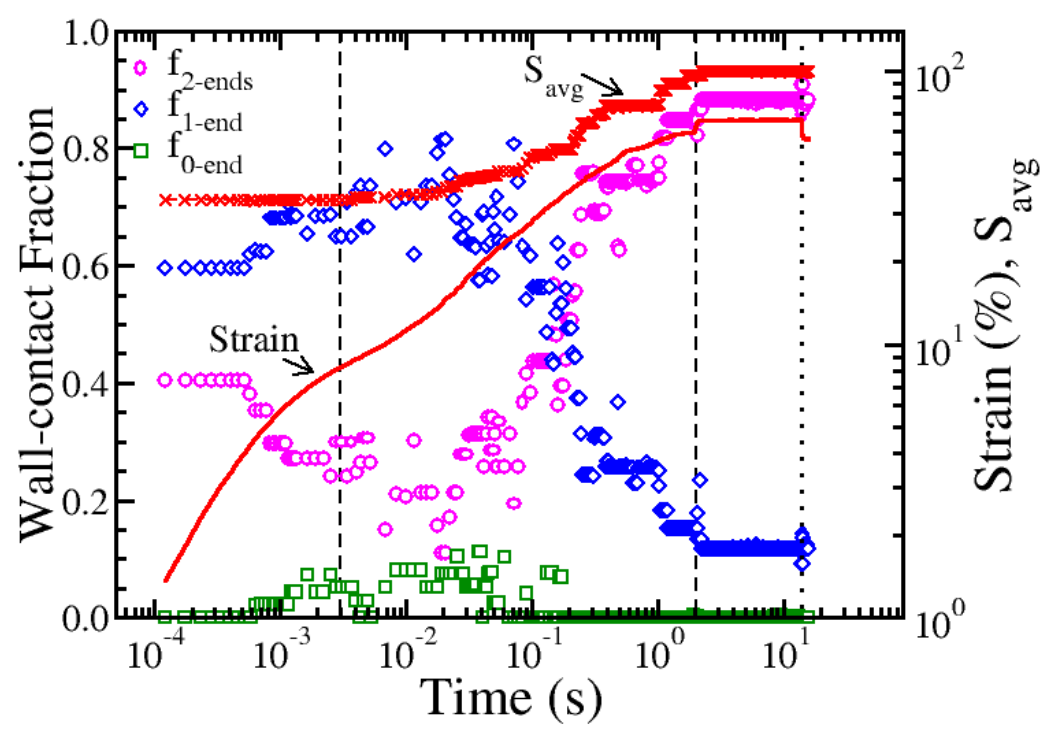


b) $\tau_{0}=250 \mathrm{~Pa}$; Preassembled chain-like structures

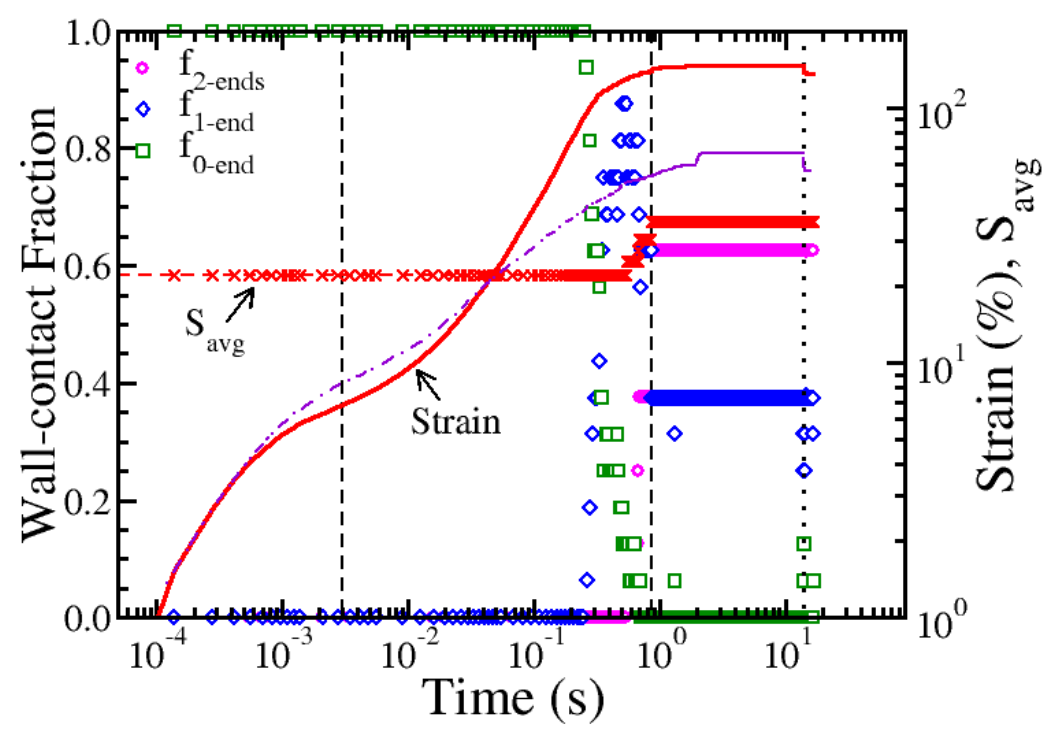

c) $\tau_{0}=1000 \mathrm{~Pa}$; Random initial configurations

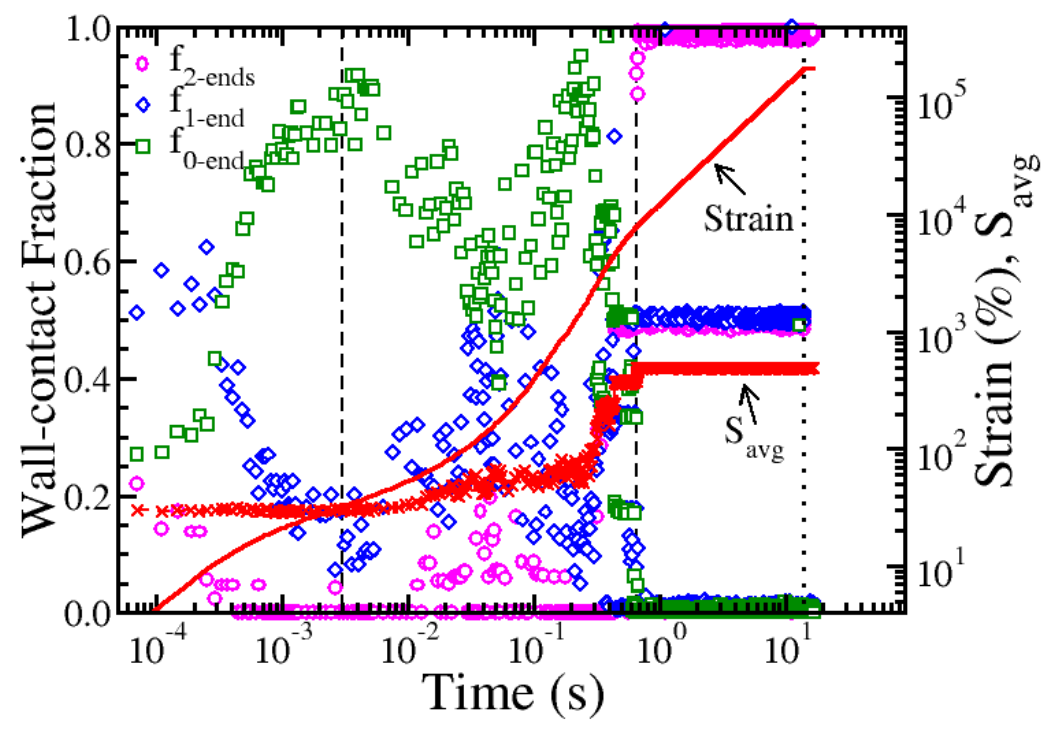


Figure 8.- Average cluster size $S_{\text {avg }}$, mean-square strength of the dipole moments $\lambda$, and fraction of particles that belong to the clusters attaching to both walls $f_{2-e n d s}$, as a function of the shear strain. The system parameters are: a) $\phi=0.05 ; H_{0}=173 \mathrm{kA} \cdot \mathrm{m}^{-1}$; $\left.\eta_{s}=20 \mathrm{mPa} \cdot \mathrm{s} ; \mathrm{b}\right) \phi=0.30 ; H_{0}=53 \mathrm{kA} \cdot \mathrm{m}^{-1} ; \eta_{s}=487 \mathrm{mPa} \cdot \mathrm{s}$.

a) $\phi=0.05$

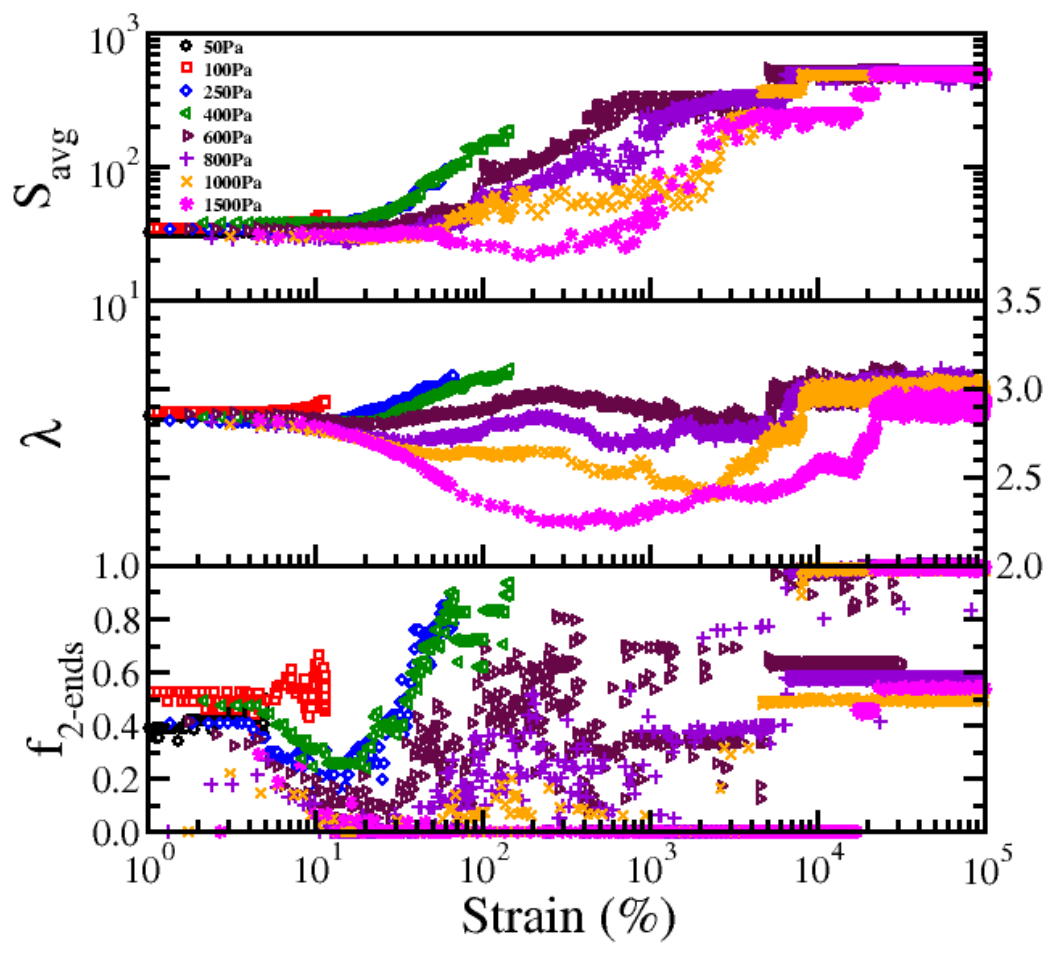


b) $\phi=0.30$

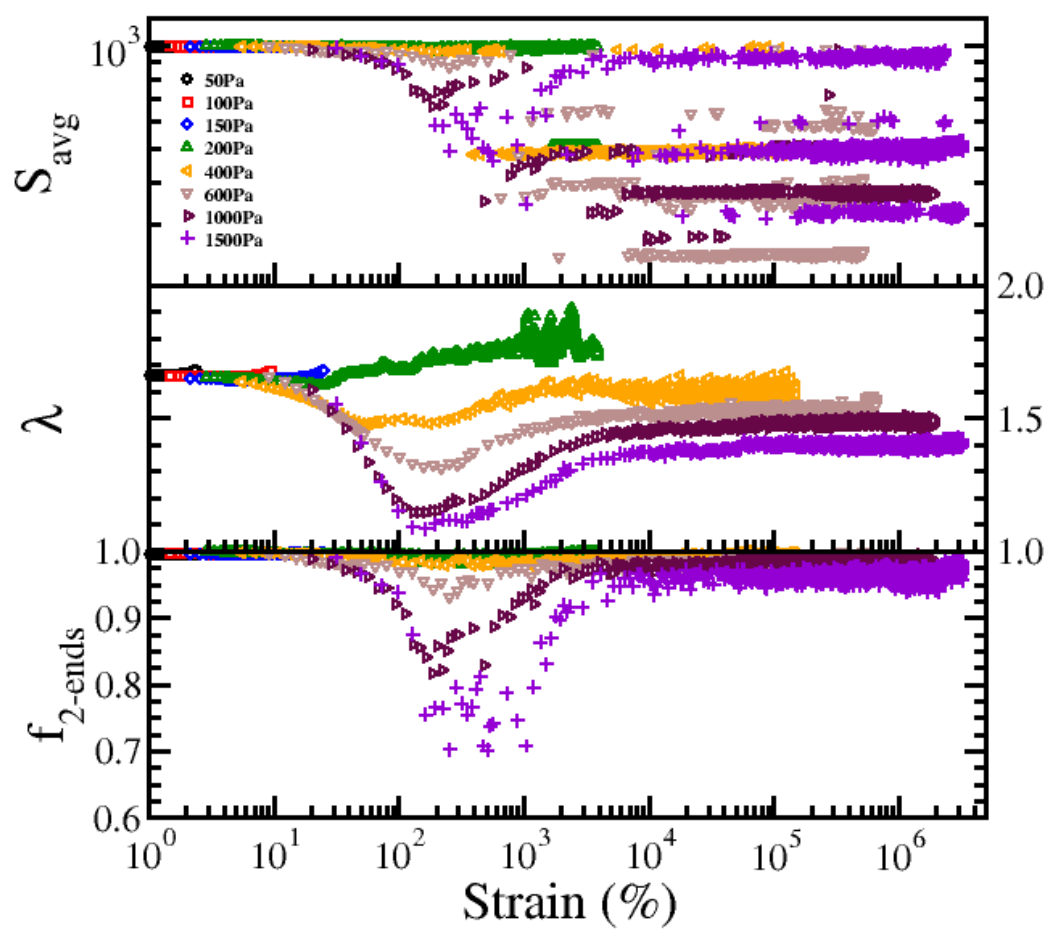


Figure 9.- Snapshots of the MR system with $\phi=0.05$ and $H_{0}=173 \mathrm{kA} \cdot \mathrm{m}^{-1}$ under applied stresses $\tau_{0}=250 \mathrm{~Pa}$ (a) and $1000 \mathrm{~Pa}$ (b and c), respectively. In a) the frames are taken at the beginning $\left(t_{\text {sim }}=0\right.$, left $)$ and end $\left(t_{\text {sim }}=14 \mathrm{~s}\right.$, right $)$ of the creep process, while in $\mathrm{b}$ ) the frames are at $t_{\text {sim }}=124.6 \mathrm{~ms}$ (left) and $t_{\text {sim }}=14 \mathrm{~s}$ (right), respectively. The configuration at $t_{\text {sim }}=0$ for the case of $\tau_{0}=1000 \mathrm{~Pa}$ (not shown) is very similar to that in the left panel of a). The snapshots in c) are the side views of one of the layers formed at $\tau_{0}=1000 \mathrm{~Pa}$, taken at time $t_{\text {sim }}=14 \mathrm{~s}$ (left) that is just before the removal of the stress and $16 \mathrm{~s}$ (right) that is in the recovery process. The simulation time scales are calculated using an effective carrier liquid viscosity $\eta_{s}^{e f f}=2.0 \mathrm{~Pa}$.s. 
a)

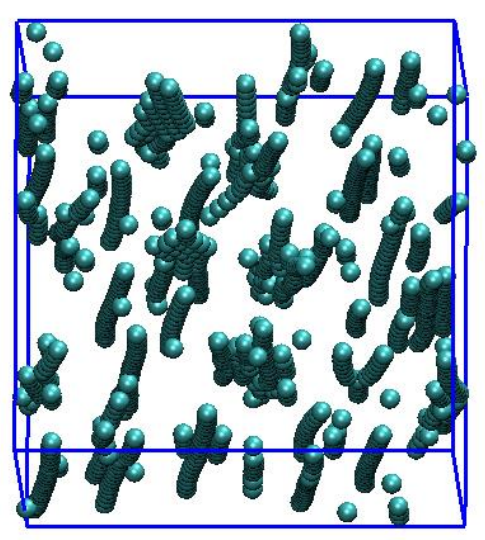

b)

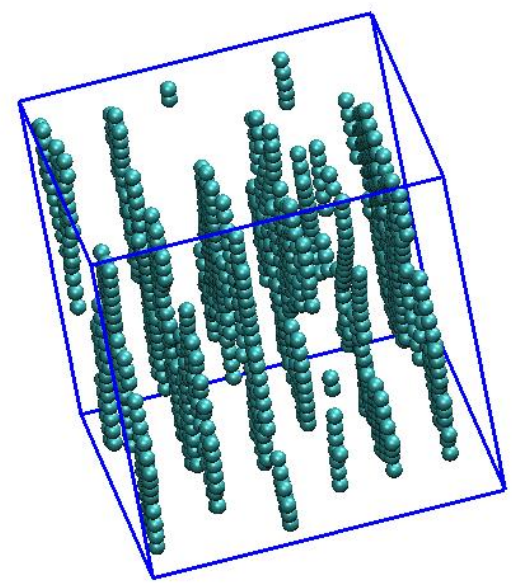

c)

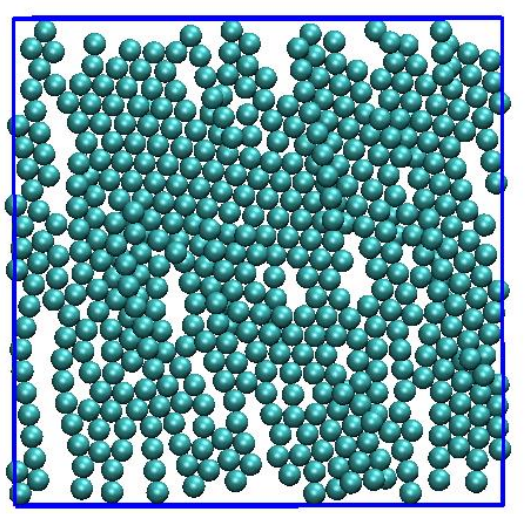

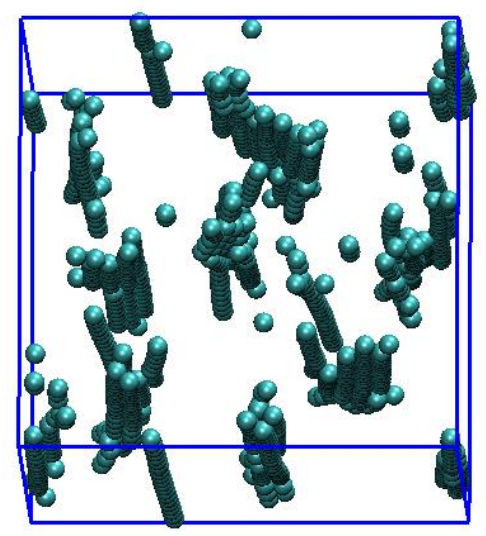
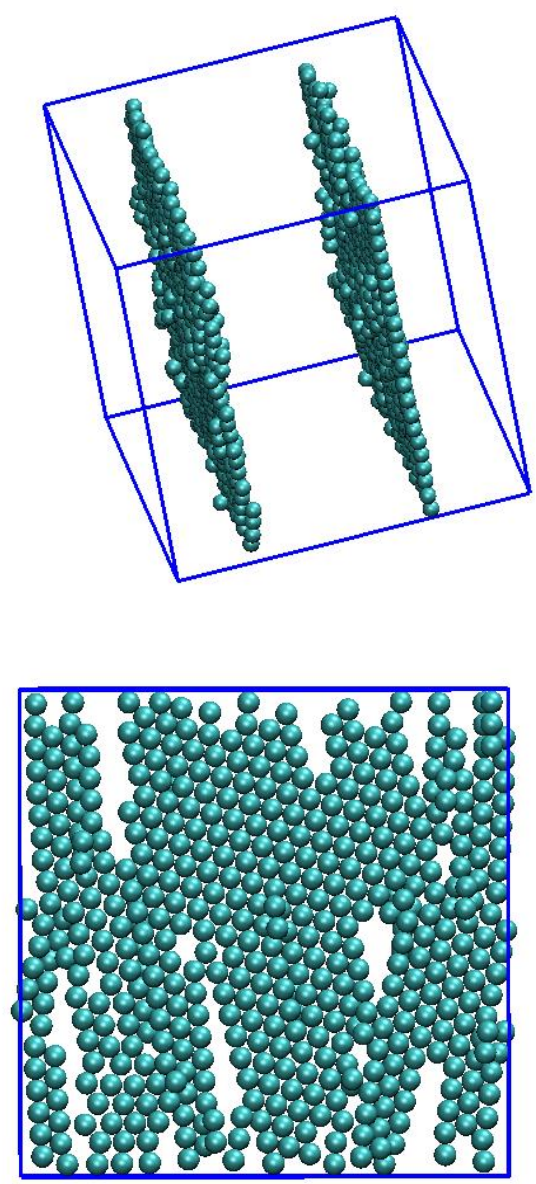
Figure 10.- Instantaneous $\left(\gamma_{i r}\right)$ and total $\left(\gamma_{i r}+\gamma_{r}\right)$ recovery strains and their ratios to the strain at the onset of the recovery process as a function of applied stress. Vertical arrows correspond to the static yield stress obtained from the extrapolation to zero shear rate of the flow curves in log-log representation, see Figure 12.

a) Experiments
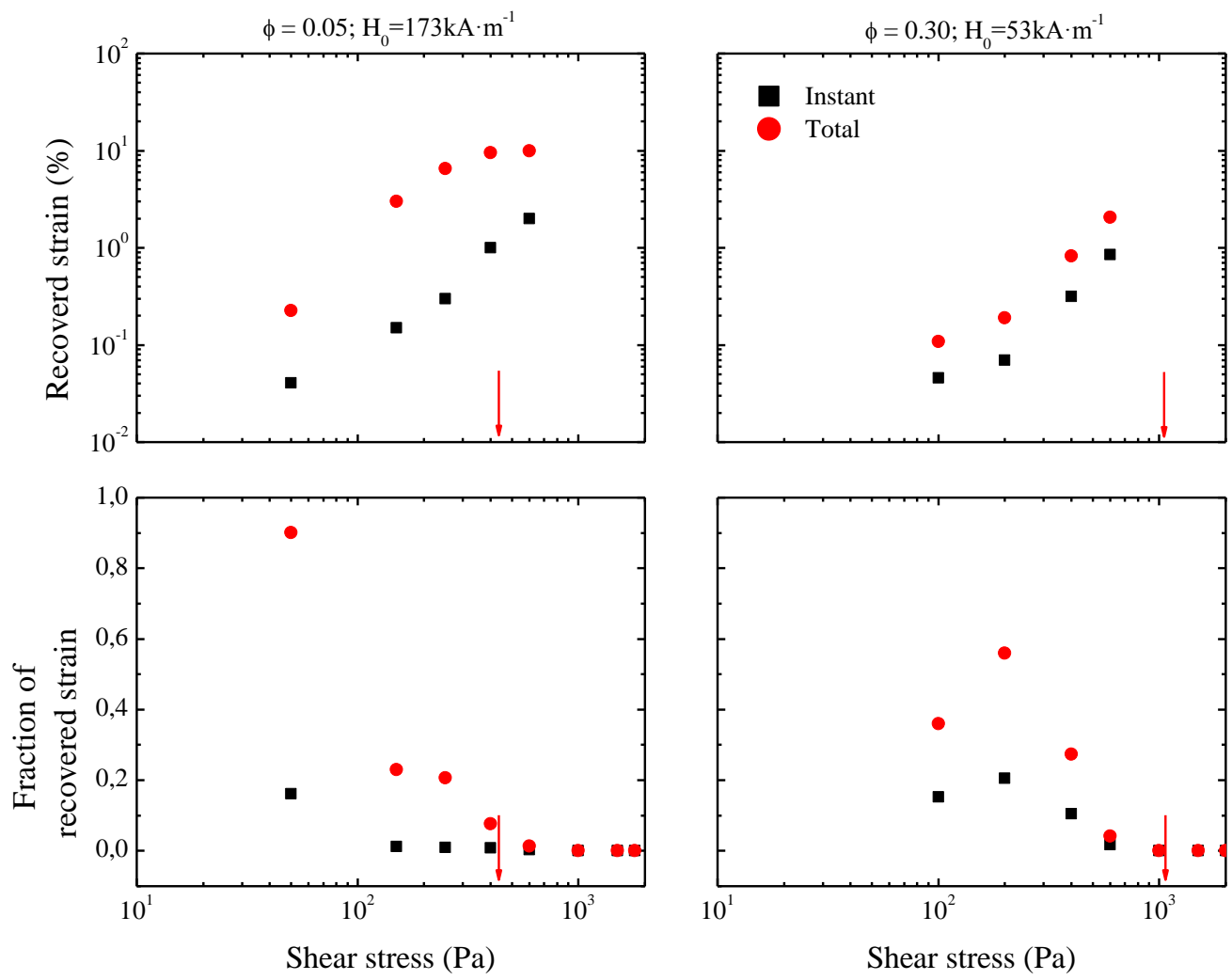
b) Simulations
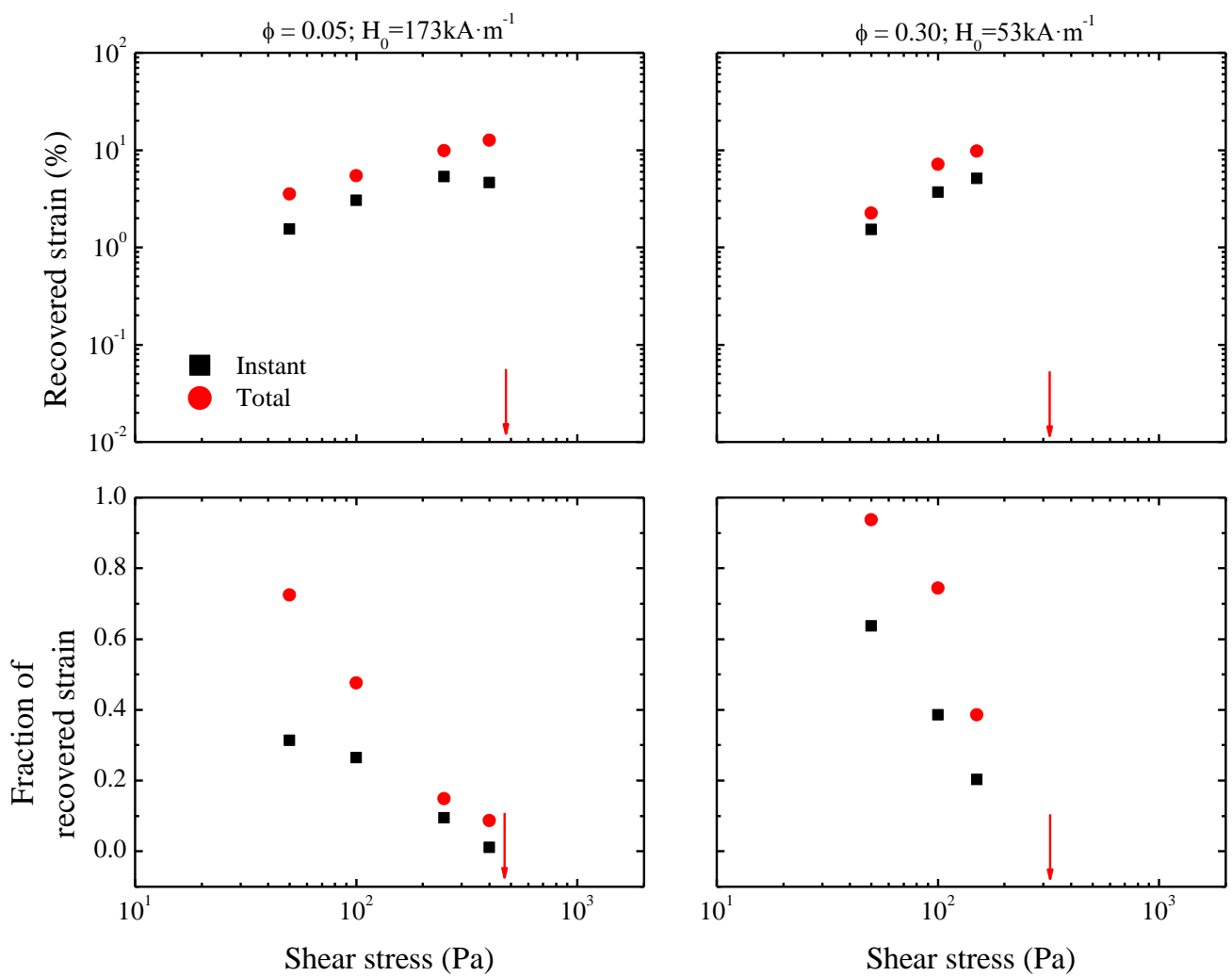
Figure 11.- Snapshots of the MR system with $\phi=0.30$ and $H_{0}=53 \mathrm{kA} \cdot \mathrm{m}^{-1}$ under applied stresses $\tau_{0}=150 \mathrm{~Pa}$ (a) and $1000 \mathrm{~Pa}$ (b), all taken at the end of the creep process. The snapshots in the left panels are the top views along the magnetic field direction, while those on the right are the side views in the $\mathrm{x}-\mathrm{z}$ (shear-field) plane. In (b) only one layer is shown where the particles form arrays aligning along the shear (horizontal) direction.

a)
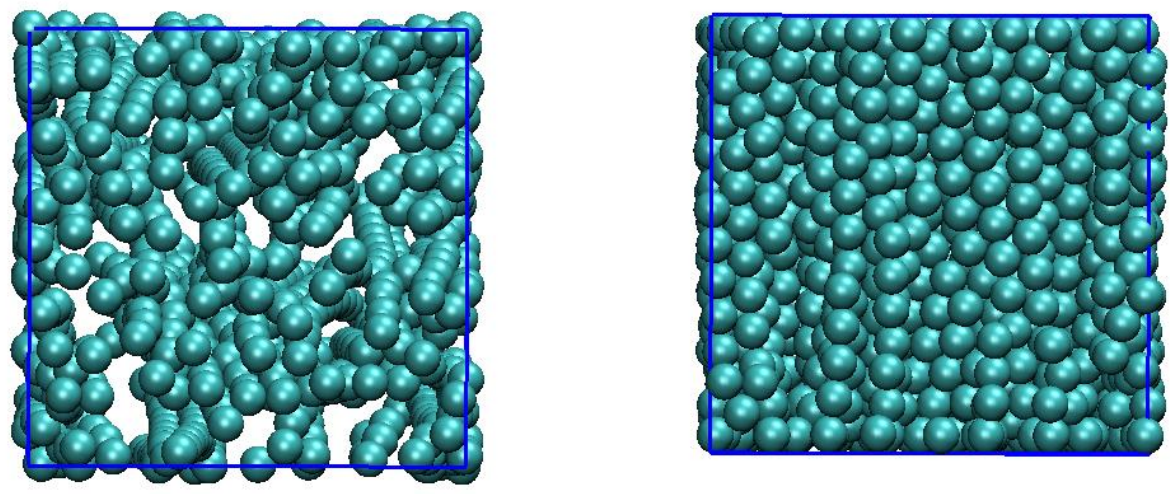

b)
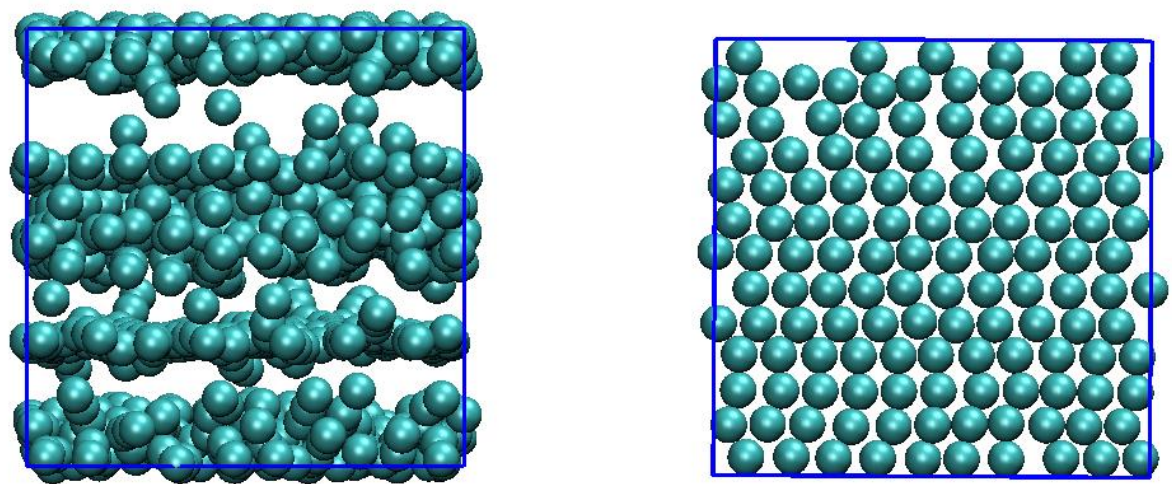
Figure 12.- Shear stress as a function of shear rate obtained from the steady-shear and creep simulations. In the steady-shear case, the contributions from the particle interactions alone, $\tau_{H}$, are also given for reference. The steady-shear experimental data are also included for comparison. The system parameters are a) $\phi=0.05, H_{0}=173$ $\mathrm{kA} \cdot \mathrm{m}^{-1}$ and silicon oil with $\eta_{s}=20 \mathrm{mPa} \cdot \mathrm{s}$; b) $\phi=0.30, H_{0}=53 \mathrm{kA} \cdot \mathrm{m}^{-1}$ and silicone oil with $\eta_{s}=487 \mathrm{mPa} \cdot \mathrm{s}$; c) $\phi=0.30, H_{0}=53 \mathrm{kA} \cdot \mathrm{m}^{-1}$ and polyalphaolefin oil with $\eta_{s}=6 \mathrm{mPa} \cdot \mathrm{s}$ without and with $(5 \mathrm{wt} \%)$ 1-octanol additive. The simulation shear rates are calculated using an effective carrier liquid viscosity $\eta_{s}^{\text {eff }}=2.0 \mathrm{~Pa} \cdot \mathrm{s}$. The dashed lines are Bingham model fittings to the experimental data points at high shear rates. 
a)

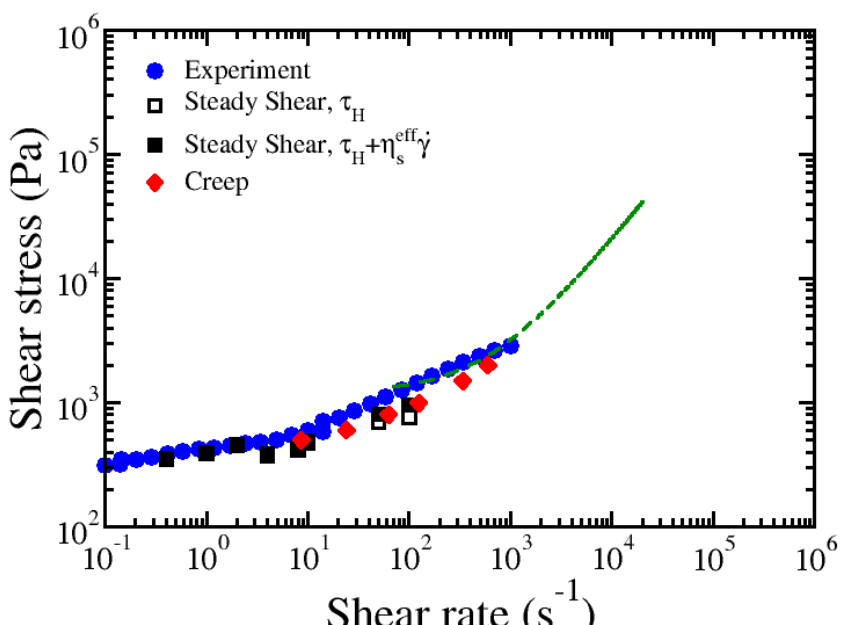

b)

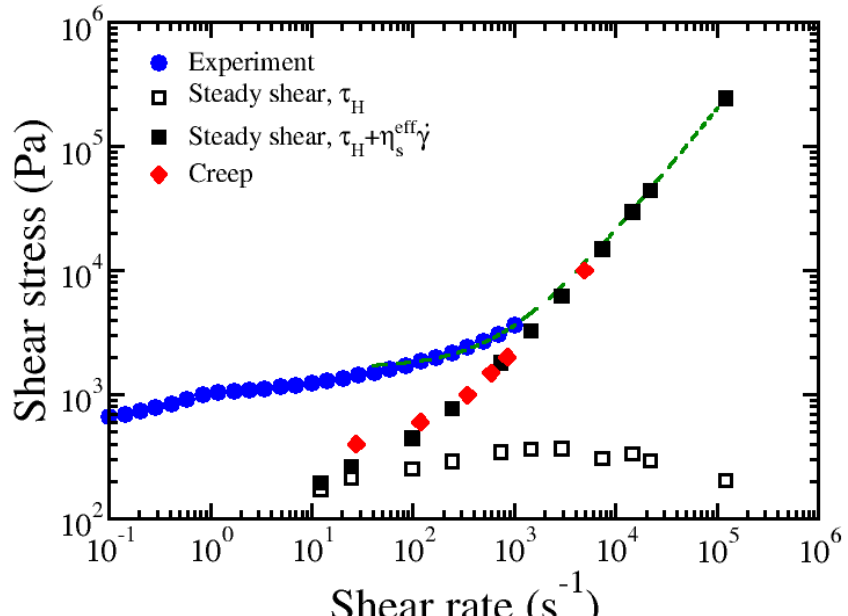

c)

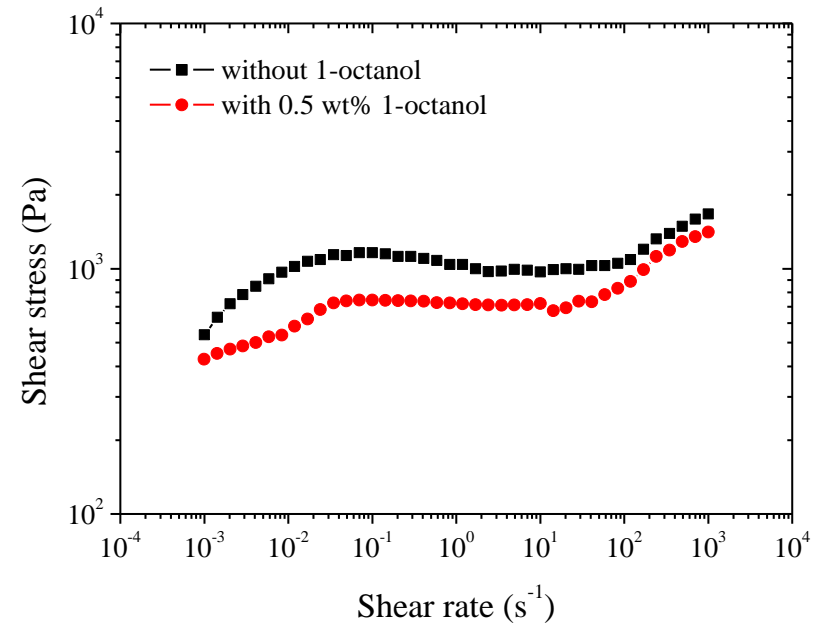




\section{SUPPLEMENTARY INFORMATION / ANNEX}

A direct comparative study between experimental and simulation results is only valid under specific circumstances. The simulation model considered in this work implies that the effect of the shear viscosity of the carrier fluid displaces the curves in the horizontal direction, strain values remaining the same whatever the viscosity value. In other words, the viscosity only appears in the time scale. Figure S1 contains experimental data for MR fluids formulated in different viscosity silicone oils employed as carriers. As observed, the trends of the curves are not affected much by the viscosity.

On the other hand, simulations are carried out at a constant shear rate. However, experimental data reported here correspond to plate-plate geometries. The choice of this particular geometry implies that the shear rate is not constant along the plate radius increasing towards the rim of the plate. In an attempt to evaluate the influence of this non-homogeneous shear profile we did run experiments on cone-plate geometries as well. Experimental results are contained in Figure S2 and demonstrate that the effect of a non-shearing flow field is not remarkable at least far from the bifurcation stress.

Finally, simulations are carried out over equilibrated structures in quiescent state. Experimentally, we include a step (c) within the protocol where the magnetic field is applied for a given (waiting) time. The waiting time chosen in this manuscript is taken to be $120 \mathrm{~s}$ and according to Figure S3 correspond to a stationary (time-independent) state. The curves shown in Figure S3 correspond to three different waiting times $(10,60$ and 120s) and all of them being very close. 
Figure S1.- Time dependence of the shear strain achieved during a step stress (creep) and recovery experiment for several stresses as indicated in the graphs. Curves correspond to MR fluids at $\phi=0.30$ prepared in silicone oils of different viscosities: $\eta_{s}=98 \mathrm{mPa} \cdot \mathrm{s}, 340 \mathrm{mPa} \cdot \mathrm{s}$, and $487 \mathrm{mPa} \cdot \mathrm{s}$. The magnetic field strength was fixed at $H_{0}=53 \mathrm{kA} \cdot \mathrm{m}^{-1}$.

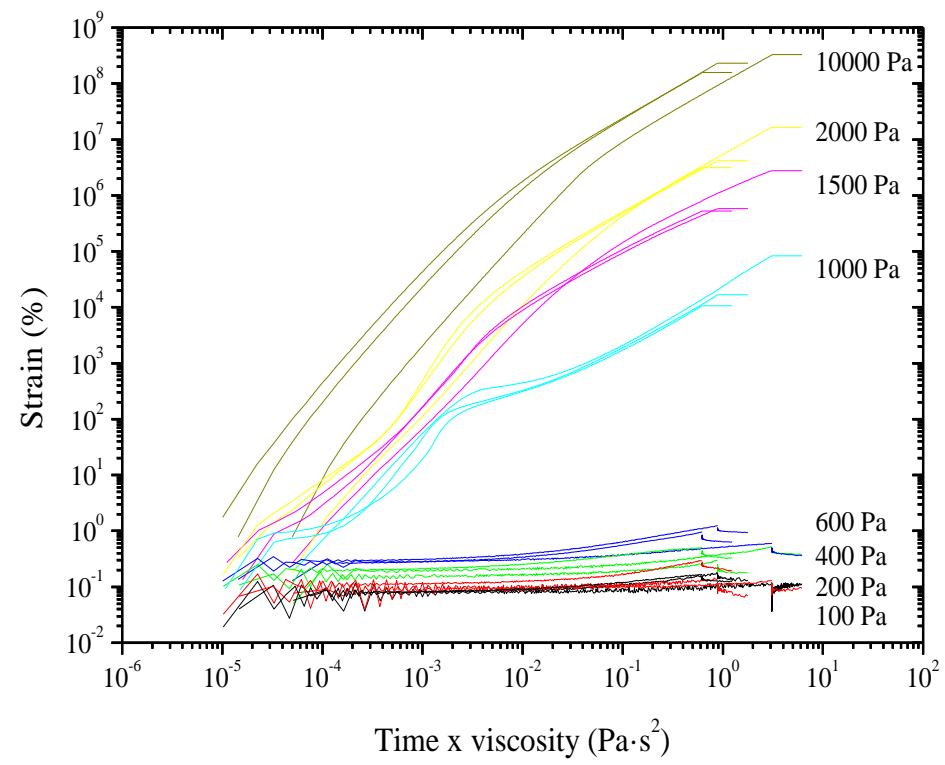


Figure S2.- Time dependence of the shear strain achieved during a step stress (creep) and recovery experiment for several stresses as indicated in the graphs. $\phi=0.30 ; H_{0}=$ $53 \mathrm{kA} \cdot \mathrm{m}^{-1} ; \eta_{s}=487 \mathrm{mPa} \cdot \mathrm{s}$. The two sets of curves correspond to plate-plate and coneplate configuration.

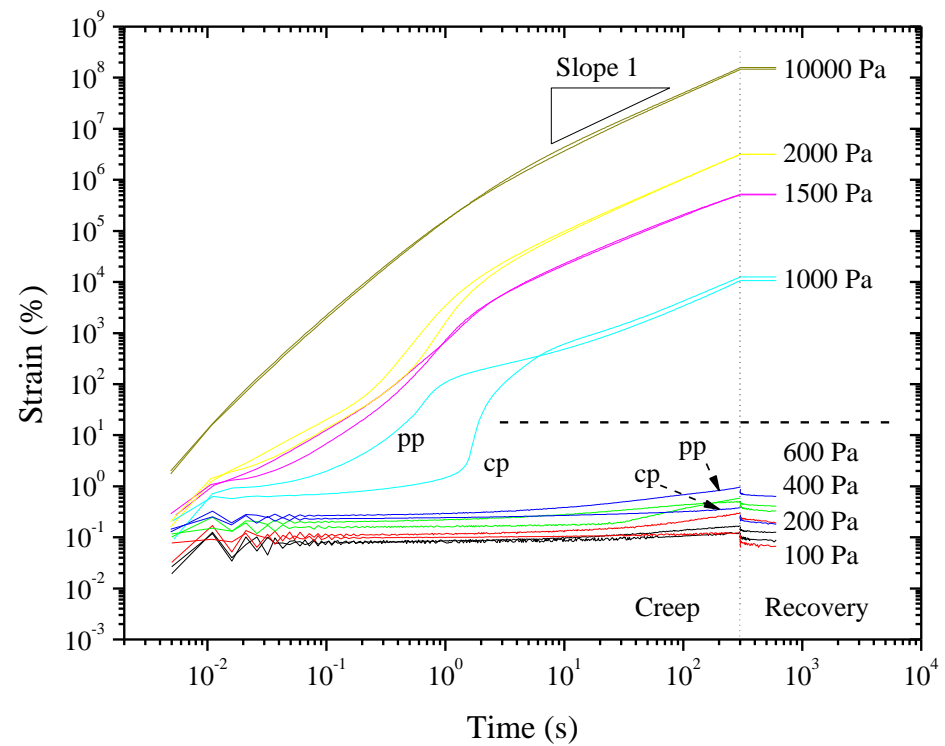


Figure S3.- Time dependence of the shear strain achieved during a step stress (creep) and recovery experiment for several stresses as indicated in the graphs. $\phi=0.30 ; H_{0}=$ $53 \mathrm{kA} \cdot \mathrm{m}^{-1} ; \eta_{s}=487 \mathrm{mPa} \cdot \mathrm{s}$. The three sets of curves correspond to three different waiting times: $10 \mathrm{~s}, 60 \mathrm{~s}$, and $120 \mathrm{~s}$.

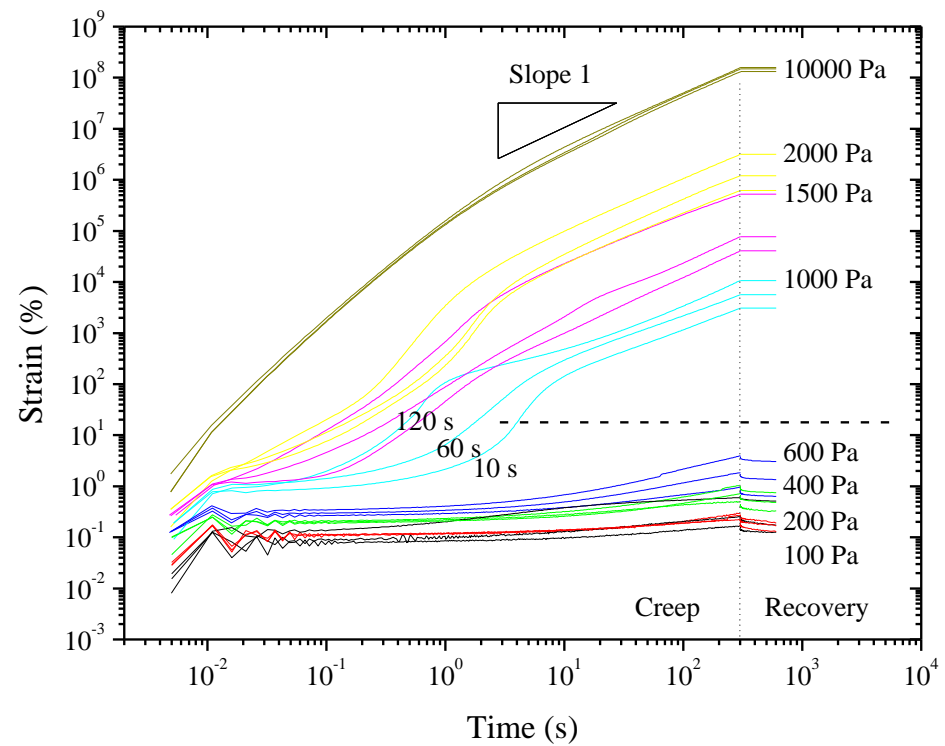

